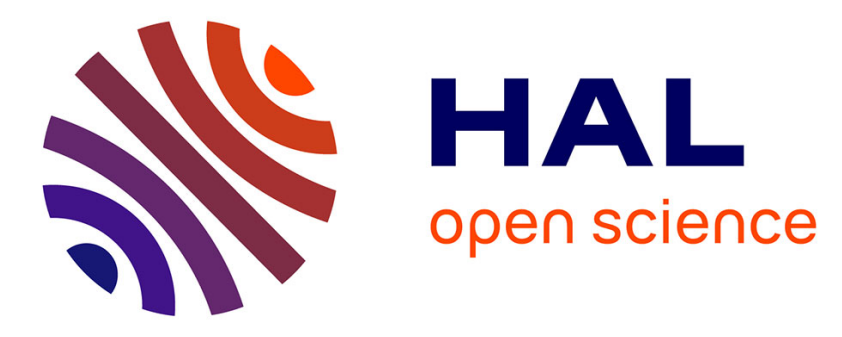

\title{
Sensory neuron-derived TAFA4 promotes macrophage tissue repair functions
}

\author{
Guillaume Hoeffel, Guilhaume Debroas, Anais Roger, Rafaëlle Rossignol, \\ Jordi Gouilly, Caroline Laprie, Lionel Chasson, Pierre Vincent Barbon, Anais \\ Balsamo, Ana Reynders, et al.
}

\section{To cite this version:}

Guillaume Hoeffel, Guilhaume Debroas, Anais Roger, Rafaëlle Rossignol, Jordi Gouilly, et al.. Sensory neuron-derived TAFA4 promotes macrophage tissue repair functions. Nature, 2021, 594 (7861), pp.9499. 10.1038/s41586-021-03563-7 . hal-03419356

\section{HAL Id: hal-03419356 \\ https://hal-amu.archives-ouvertes.fr/hal-03419356}

Submitted on 8 Nov 2021

HAL is a multi-disciplinary open access archive for the deposit and dissemination of scientific research documents, whether they are published or not. The documents may come from teaching and research institutions in France or abroad, or from public or private research centers.
L'archive ouverte pluridisciplinaire HAL, est destinée au dépôt et à la diffusion de documents scientifiques de niveau recherche, publiés ou non, émanant des établissements d'enseignement et de recherche français ou étrangers, des laboratoires publics ou privés. 
2 Sensory neuron-derived TAFA4 promotes macrophage tissue repair

\section{functions}

4

5

6 Guillaume Hoeffel $^{1 * \#}$, Guilhaume Debroas ${ }^{1 *}$, Anais Roger ${ }^{1}$, Rafaelle Rossignol $^{1}$, Jordi

7 Gouilly $^{1}$, Caroline Laprie ${ }^{1}$, Lionel Chasson ${ }^{1}$, Pierre Vincent Barbon ${ }^{1}$, Anais Balsamo ${ }^{1}$,

8 Ana Reynders ${ }^{2}$, Aziz Moqrich ${ }^{2}$ and Sophie Ugolini ${ }^{1 \#}$

9

10

$11{ }^{1}$ Aix Marseille Univ, CNRS, INSERM, CIML, Centre d'Immunologie de Marseille12 Luminy, Marseille, France

$13{ }^{2}$ Aix Marseille Univ, CNRS, IBDM, Institut de Biologie du Développement de 14 Marseille, Marseille, France

$15 \quad$ *Equal contributions

16 \#Corresponding authors: hoeffel@ciml.univ-mrs.fr; ugolini@ciml.univ-mrs.fr

17

18

19 Keywords: Monocytes, macrophages, sensory neurons, neuropeptides, tissue repair,

20 skin fibrosis, TAFA4, GINIP

21

22 Running title: TAFA4 triggers IL-10 production by dermal macrophages and promotes

23 skin repair

24 
Inflammation is a defense response to tissue damage requiring tight regulation to prevent impaired healing. Tissue-resident macrophages play a key role in tissue repair $^{1}$ but the precise molecular mechanisms regulating the balance between inflammatory and pro-repair macrophage responses during healing remain poorly understood. We demonstrate here a major role of sensory neurons in promoting macrophage tissue repair functions. In a sunburn-like model of skin damage, the conditional ablation of sensory neurons expressing the Gaiinteracting protein (GINIP) resulted in defective tissue regeneration, and dermal fibrosis. Dissection of the underlying molecular mechanisms revealed a crucial role for the neuropeptide TAFA4, produced in the skin by C-low threshold mechanoreceptors (C-LTMR), a subset of GINIP ${ }^{+}$neurons. TAFA4 modulated the inflammatory profile of macrophages directly in vitro. In vivo analyses in Tafa4deficient mice revealed that TAFA4 promoted IL-10 production by dermal macrophages after UV-induced skin damage. This TAFA4/IL-10 axis also ensured the survival and maintenance of $\mathrm{IL}_{-10}{ }^{+} \mathrm{Tim} 4^{+}$dermal macrophages, resolving skin tissue damage, creating new therapeutic perspectives for inflammatory diseases. 
51 Skin overexposure to ultraviolet (UV) light causes sunburn, characterized by epidermis

52 destruction and inflammation of the underlying dermal papilla ${ }^{2}$. Such tissue damage

53 induces a complex inflammatory response, requiring tight regulation to prevent 54 persistent injury. Sunburn is also characterized by a transient phase of painful 55 hypersensitivity, mediated by the activation of specialized skin-innervating sensory neurons $s^{3,4}$. Studies of other pathological cutaneous conditions have shown that the peripheral nervous system regulates cutaneous inflammatory processes ${ }^{3,5-9}$. Most studies have focused on the roles of TRPV $1^{+}$peptidergic primary sensory neurons and the neuropeptide CGRP ${ }^{3,5-7,9}$ (calcitonin gene-related peptide). However, the potential immunoregulatory role of non-peptidergic C-fibers expressing GINIP ${ }^{10}$ remains

61 unknown.

\section{GINIP $^{+}$neurons prevent $U$ V-induced skin fibrosis}

We analyzed the role of somatosensory neurons in tissue repair in mouse ears exposed to UV-C radiation, an experimental model of sunburn (Fig. 1a). We assessed the expression of genes associated with tissue inflammation and repair in skin from wildtype (WT) mice, following UV overexposure. Genes encoding inflammatory molecules, such as $I l-1 \beta$ and $\operatorname{Tnf} \alpha$, were upregulated until day 7 post-UV irradiation

69 (pi), whereas pro-repair genes, such as Collal and Retlna, were upregulated from day

7014 until day 35 pi, defining the resolution/repair phase of this model (Extended data

\section{$71 \quad$ Fig. 1a).}

72 We analyzed the consequences for the somatosensory nervous system of skin 73 overexposure to UV light. Most of the neurons responsible for detecting noxious stimuli 74 and eliciting pain perception express the sodium channel Nav1.8 $8^{11-13}$, and have cell 
bodies in the dorsal root ganglia (DRGs) or trigeminal ganglion. We monitored the expression of ATF3 (Activating Transcription Factor 3), a transcription factor used as a proxy for neuronal injury and activation ${ }^{14}$, in DRGs after skin exposure to UV. Atf3 expression significantly increased in C2/C3 DRGs, which specifically innervate ear skin (Fig. 1b, Extended data Fig. 1b-d).

We determined the phenotype of $\mathrm{ATF}^{+} \mathrm{DRG}$ neurons, by immunofluorescence staining. Very few of these neurons co-expressed CGRP pi, whereas up to $50 \%$ coexpressed GINIP (Fig. 1c, d, Extended data Figure 1e, f), suggesting that UV-induced skin inflammation essentially affects GINIP $^{+}$somatosensory neurons.

We therefore investigated the role of these Nav1. $8^{+} \mathrm{GINIP}^{+}$neurons $\left(\mathrm{GINIP}^{+}\right.$neurons) in tissue repair, by conditional depletion in the GINIP-DTR mode ${ }^{10,15}$ (Extended data Fig. 2a-c ). Between days 5 and 35 pi, ear skin was significantly thicker in GINIP-DTR mice than in control DTR ("control") littermates (Fig. 1e). Macroscopic analysis also revealed greater redness, tissue shrinkage and signs of necrosis leading to ear tissue loss in GINIP-DTR mice (Fig. 1f). These skin lesions were not associated with an increase in scratching in GINIP-DTR mice (Extended data Fig. 2d). Histological analysis showed similar steady-state skin structures in GINIP-DTR and control littermates. By contrast, 35 days pi, GINIP-DTR mice displayed marked pinna thickening, with an increase in horizontally oriented collagen fiber deposition, persistent leukocytic infiltration and focal auricular cartilage obliteration, whereas control mice presented signs of skin healing (Fig. 1g-j, Extended data Fig. 2e-j). Thus, GINIP ${ }^{+}$neurons prevent skin over-inflammation and fibrosis after UV irradiation.

\section{Impaired macrophage response in GINIP-DTR mice}


99 We investigated whether the profound tissue repair defect of GINIP-DTR mice was 100 linked to dysregulation of the cutaneous immune response. The absolute numbers of 101 dendritic cells (DC), Langerhans cells (LC), mast cells, lymphoid cells and 102 granulocytes were similar in GINIP-DTR and control mice, at steady state and 14 days 103 pi, whereas GINIP-DTR mice had fewer monocytes/macrophages $(\mathrm{Mo} / \mathrm{M} \phi)$ than 104 control mice pi (Extended data Fig. 3a-d). None of these immune cells expressed DTR 105 in GINIP-DTR mice, and their numbers were unaffected by DT treatment in 106 homeostatic conditions (Extended data Fig. 3b-d, f).

107 Macrophages regulate all stages of tissue repair ${ }^{1}$, and dysregulation of their homeostasis 108 can lead to chronic inflammation, excessive collagen deposition and 109 hypertrophic/fibrotic scars ${ }^{1}$. Dermal resident M $\phi$ expressing CD206 were observed 110 near GINIP ${ }^{+}$nerve terminals, suggesting possible functional interaction (Fig. 2a-b, 111 Extended data Fig. 3g). We analyzed the dermal Mo/M $\phi$ response upon UV 112 overexposure. Tissue-resident $\mathrm{M} \phi$ emerge either during embryonic development ${ }^{16-18}$ or 113 differentiate postnatally from circulating monocytes ${ }^{18,19}$. Upon inflammation, 114 monocyte subsets ${ }^{20}$, including “classical” Ly6C $\mathrm{C}^{+}$monocytes (Mo), "patrolling” Ly6C $\mathrm{C}^{\text {lo }}$ 115 monocytes (PMo) and "intermediate" Ly6C $\mathrm{C}^{+} \mathrm{HC}-\mathrm{II}^{+}$monocytes (Int. Mo), can 116 generate short-lived MHC-II ${ }^{+}$monocyte-derived DC (MoDC) and monocyte-derived $117 \mathrm{CD}^{+}{ }^{+} \mathrm{CD} 206^{-}$inflammatory macrophages (Inflam $\left.\mathrm{M} \phi\right)$. We identified eight $\mathrm{Mo} / \mathrm{M} \phi$ 118 subsets based on differential expression of CCR2, CD64, Ly6C, MHC-II, CD206 and 119 Tim4 (Fig. 2c, Extended data Fig. 4a-c). Their steady-state distributions were similar 120 in the skin of GINIP-DTR and control mice (Fig. 2e, f, Extended data Fig. 4d-h). By 121 contrast, absolute numbers of $\mathrm{Tim} 4^{+}$dermal $\mathrm{M} \phi$ were very low from days 3 to 14 pi in 122 the skin of GINIP-DTR mice (Fig. 2c-e), whereas Ly6C ${ }^{+}$Mo recruitment increased on 123 day 3 pi (Extended data Fig. 4d). This influx of $\mathrm{Ly}^{+} \mathrm{C}^{+} \mathrm{Mo}$ was associated with 
increases in Inflam M $\phi$ numbers in GINIP-DTR mice from days 3 to 14 pi (Fig. 2f),

125 consistent with the stronger skin inflammation observed in these mice (Fig. 1e). By

126 contrast, the expansion of DN and MHC-II ${ }^{+}$dermal M $\phi$ subsets was little affected by

127 the absence of GINIP ${ }^{+}$neurons (Extended data Fig. 4e-h). Thus, signals from GINIP ${ }^{+}$

128 sensory neurons promote $\mathrm{Tim}^{+}$dermal $\mathrm{M} \phi$ maintenance and control Inflam $\mathrm{M} \phi$

129 responses following UV-induced skin damage.

\section{TAFA4 promotes skin repair upon UV exposure}

132 We investigated the mechanisms underlying the immunomodulatory and pro-repair role 133 of $\mathrm{GINIP}^{+}$neurons. GINIP is expressed by two somatosensory neuron subsets: $134 \mathrm{GINIP}^{+} / \mathrm{IB}^{+}$neurons, which also express MRGPRD and selectively innervate the 135 epidermis, and $\mathrm{GINIP}^{+} / \mathrm{IB}^{-}$neurons, which express TAFA4, TH and VGLUT3, 136 markers of the C-low-threshold mechanoreceptors (C-LTMRs) selectively innervating 137 hair follicles ${ }^{10,21}$ (Extended data Fig. 5a-c). Binding to isolectin-B4 (IB4) and TAFA4 138 can be used to distinguish between these subsets in DRGs ${ }^{10,22,23}$. We generated anti139 TAFA4 monoclonal antibodies (Extended data Fig. 5d, g), for C-LTMR 140 identification. As expected, $\mathrm{TAFA} 4^{+}, \mathrm{TH}^{+}$and $\mathrm{IB}^{+}$neurons were ablated in GINIP141 DTR mice, whereas $\mathrm{CGRP}^{+}$neurons were spared (Extended data Fig. 2b). DRG 142 staining showed that $10-20 \%$ of $\mathrm{ATF}^{+}$neurons were $\mathrm{IB}^{+}$and $20-50 \%$ were $\mathrm{TAFA} 4^{+}$

143 (Fig. 3a, b, Extended data Fig. 5e, f), suggesting potential roles for these subsets of 144 GINIP $^{+}$neurons in regulating UV-induced skin inflammation.

145 C-LTMRs mediate two opposing aspects of touch sensation: pleasantness and injury146 induced mechanical pain ${ }^{10,22}$. TAFA4 is a secreted protein ${ }^{24}$ that can modulate injury147 induced mechanical hypersensitivity and chemical pain in mice ${ }^{22}$. TAFA4 promotes 
148 human macrophage chemotaxis in vitro ${ }^{25}$, but its impact on myeloid cell responses in 149 vivo remains unknown.

150 We established an ELISA for TAFA4 detection in tissues. UV exposure induced 151 increases in TAFA4 levels in WT mouse DRGs and skin (Fig. 3c, Extended data Fig. $152 \mathbf{5 h})$. We investigated the cellular source of TAFA4 in skin. Consistent with publicly 153 available database (http://www.immgen.org; http://biogps.org), Tafa4 (Fam19a4) 154 transcription was observed in DRGs, but not in the blood, gut, lung or skin (Extended 155 data Fig. 5i). Tafa4 transcription has been reported in human M $\phi$ following LPS 156 stimulation ${ }^{25}$. However, we detected no Tafa4 transcripts in BM-derived macrophages 157 (BMDM) activated with LPS (+/-TAFA4) or in sorted CD206 ${ }^{+}$dermal M $\phi$ on days 0 158 and 7 pi (Extended data Fig. 5j). Tafa4 mRNA was undetectable in the skin, even after 159 UV exposure, but abundant in DRGs pi (Extended data Fig.1a, 5k). These data 160 strongly suggest that Tafa4 is transcribed only in DRG neuron cell bodies, with protein 161 release into the skin via C-LTMR nerve endings.

162 We investigated the possible role of TAFA4 in regulating tissue regeneration pi, by 163 studying Tafa4-KO mice ${ }^{22}$. From days 14 to 35 pi, Tafa-4-KO mice had thicker ear skin 164 than control littermates, suggesting defective resolution of skin inflammation in the 165 absence of TAFA4 (Extended data Fig. 6a). No histological change in the skin of 166 Tafa4-KO mice was observed in homeostatic conditions. By contrast, 35 days pi, 167 leukocyte infiltration score and epidermal thickness were higher in Tafa4-KO mice, 168 which displayed more extensive, persistent dermal fibrosis (Extended data Fig. 6b, c). 169 Picro Sirius Red staining revealed characteristic excessive type 1 collagen deposition 170 in unresolved fibrotic scars in the dermis of Tafa4-KO mice (Fig. 3d, e). Thus, like 171 GINIP-DTR mice, Tafa4-KO mice presented pathological fibrosis, suggesting a 172 requirement for the neuropeptide TAFA4, produced by C-LTMRs, for skin repair pi. 
TAFA4 regulates dermal macrophage IL-10 production

175 We investigated the mechanisms by which TAFA4 regulates skin repair. We monitored 176 inflammatory cytokine and chemokine concentrations in skin from Tafa4-KO mice and 177 control littermates from days 0 to 35 pi. TNF $\alpha$, IL-1 $\beta$, IL-6, CCL2, CCL4 and CXCL1 178 (Fig. 3f, Extended data Fig. 6d) levels decreased between days 7 and 10 pi in control 179 mice, but remained higher in Tafa4-KO mice. By contrast, expression levels of the anti180 inflammatory cytokine IL-10 were lower in the skin of Tafa4-KO mice pi (Fig. 3g, 181 Extended data Fig. 6d). Resolution of the inflammatory phase was therefore impaired 182 in the absence of TAFA4.

183 IL-10 is a potent anti-inflammatory molecule. We hypothesized that impaired IL-10 184 production might underlie the observed dysregulation of skin repair in UV-exposed 185 Tafa4-KO mice. Intracellular staining and analyses in IL-10-GFP reporter mice 186 identified resident $\mathrm{M} \phi$ and mast cells ${ }^{26}$ as the main producers of IL-10, with regulatory 187 T cells (Tregs) producing only small amounts (Extended data Fig. 6e-i). Mast cell 188 numbers and IL-10 production decreased strongly on day 3 pi (Extended data Fig. 6j189 m). TAFA4 deficiency was not associated with modulation of IL-10 production by 190 Tregs and mast cells (Extended data Fig. 6i-m). By contrast, Ill0 levels in sorted $191 \mathrm{CD}^{206^{+}}$dermal M $\phi$ were lower in Tafa4-KO mice than in controls on days 7 and $10 \mathrm{pi}$ 192 (Fig. 3h). Intracellular staining confirmed that TAFA4 was required for the 193 upregulation of IL-10 production in all CD206 ${ }^{+} \mathrm{M} \phi$ subsets after UV overexposure, 194 with $\mathrm{Tim}^{+} \mathrm{M} \phi$ producing more IL-10 than DN and $\mathrm{MHC}-\mathrm{II}^{+} \mathrm{M} \phi$ (Extended data Fig. 195 7a-c).

196 We investigated whether TAFA4 regulated M $\phi$ activation directly. BMDM or purified 197 peritoneal $\mathrm{M} \phi(\mathrm{ThioM} \phi)$ were activated with LPS in the presence or absence of TAFA4. 
$\operatorname{Tnf} \alpha, I l 1 \beta$ and Il6 transcripts were significantly downregulated, whereas $I l 10$

199 transcripts were upregulated by TAFA4 (Fig. 3i, Extended data Fig.7e). Together, 200 these data suggest that the TAFA4-mediated regulation of IL-10 production in the skin 201 upon UV-exposure is mediated essentially through the regulation of dermal $202 \mathrm{M} \phi$ functions.

203 Formyl peptide receptor 1 (FPR1) has been described as a TAFA4 receptor inducing 204 the chemotaxis of human $\mathrm{M} \phi$ and transfected cell lines in vitro ${ }^{25}$. We observed no 205 chemotactic effect of TAFA4 on mouse BMDM or isolated peritoneal $\mathrm{M} \phi$ in vitro 206 (Extended data Fig. 7d and data not shown). Consistently in vivo, monocyte-derived 207 cell recruitment pi was not reduced in GINIP-DTR mice (Extended data Figure 4d). 208 Moreover, TAFA4 had a similar immunoregulatory effect on BMDM from FPR1-KO 209 and WT mice showing that at least one other unknown receptor drives the functional 210 effect of TAFA4 on M $\phi$ (Extended data Fig. 7f). Thus, TAFA4 can regulate the 211 inflammatory profile of $\mathrm{M} \phi$ directly, in an FPR1-independent manner. It will be 212 important to determine whether macrophage regulation by TAFA4 differs between 213 mice and humans.

\section{A TAFA4-IL10-Tim4 ${ }^{+}$macrophage axis promotes tissue repair}

216 We then investigated whether TAFA4 affected the dynamics between dermal resident

$217 \mathrm{M} \phi$ and newly recruited monocyte-derived cells during repair. We first performed a 218 lineage-tracing analysis, tracking the fate of $\mathrm{CX} 3 \mathrm{CR} 1^{+}$embryonic $\mathrm{M} \phi$ precursors using 219 the tamoxifen-dependent CX3CR1 $1^{\text {CreERT2 }}$ :R26-YFP mouse model ${ }^{27}$. YFP expression 220 was selectively induced in $\mathrm{CX} 3 \mathrm{CR} 1^{+}$cells on day 16.5 of embryonic development. In 221 adult mice under homeostatic conditions, the $\mathrm{MHC}-\mathrm{II}^{+}$and $\mathrm{DN}$ dermal $\mathrm{M} \phi$ subsets only 222 weakly expressed YFP (Extended data Fig. 8a, b). By contrast, 20\% of Tim $4^{+} \mathrm{M} \phi$ 
were $\mathrm{YFP}^{+}$, like $\mathrm{LCs}^{28}$, suggesting that $\mathrm{Tim}^{+} \mathrm{M} \phi$ are mostly bona fide embryo-derived

224 cells.

225 We then assessed the contribution of monocytes to the various dermal Mo/M $\phi$ subsets,

226 by generating shield-irradiated BM chimeras (Extended data Fig. 8c). As expected,

227 two months after CD45.1 BM reconstitution in CD45.2 recipients, 90\% of blood

228 monocytes were CD45.1 $1^{+}$, whereas microglia were entirely of recipient $\left(\mathrm{CD} 45.2^{+}\right)$

229 origin. Under homeostatic conditions, dermal Ly6C ${ }^{+}$Mo, Int Mo, PMo, MoDC and 230 Inflam $\mathrm{M} \phi$ were mostly $\mathrm{CD} 45.1^{+}$, confirming their $\mathrm{BM}$ origin, whereas $\mathrm{Tim}^{+} \mathrm{M} \phi$

231 were CD45.2 $2^{+}$, demonstrating their self-maintenance over time independently of 232 circulating monocytes (Extended data Fig. 8d). However, 120 days pi, the CD45.1 233 chimerism of DN and Tim4 M $\phi$ increased (Extended data Fig. 8e), showing that 234 monocytes recruited pi acquired a resident-like $\mathrm{M} \phi$ phenotype with self-renewing 235 capacity.

236 We then generated shield-irradiated BM chimeras, using WT or Tafa4-KO (both 237 CD45.2) recipient mice (Fig. 4a-f, Extended data Fig. 8f-h). Intracellular staining on 238 day 7 pi revealed that IL-1 $\beta$ expression was mostly associated with monocyte-derived 239 cells, whereas TNF $\alpha$ expression was restricted essentially to Inflam $\mathrm{M} \phi$ (Fig. 4c). The 240 number of Inflam M $\phi$ cells in Tafa4-KO chimeric mice was higher than that in control 241 chimeras, suggesting that TAFA4 reduces the number of newly derived Inflam $\mathrm{M} \phi$ 242 (Fig. 4d). Tim $^{+} \mathrm{M} \phi$ were the predominant CD45.2 $2^{+}$population in both chimeras (Fig. $243 \mathbf{4 b}, \mathbf{c})$, consistent with our previous analysis. Tim $4^{+} \mathrm{M} \phi$ produced large amounts of IL24410 , but no IL-1 $\beta$ or TNF $\alpha$ (Fig. 4c), highlighting their anti-inflammatory function. 245 TAFA4 deficiency had little effect on DN and MHC-II ${ }^{+} \mathrm{M} \phi$ homeostasis pi (Extended 246 data Fig. 8h). By contrast, the absolute number of $\mathrm{CD} 45.2^{+} \mathrm{Tim}^{+} \mathrm{M} \phi$, which was 
similar in both chimeras under homeostatic conditions, strongly decreased in Tafa4-

$248 \mathrm{KO}$ recipient mice pi (Fig. 4e left). Overall CD45.1 chimerism levels in $\mathrm{Tim}^{+} \mathrm{M} \phi$

249 revealed that $\mathrm{CD} 45.2^{+} \mathrm{Tim} 4^{+} \mathrm{M} \phi$ were replaced by $\mathrm{CD} 45.1^{+}$monocyte differentiation

250 to a greater extent in Tafa4-KO than in WT recipients (Fig. 4e right). However, the

251 overall $\mathrm{Tim}^{+} \mathrm{M} \phi$ pool expanded to a lesser extent in the absence of TAFA4 (Fig. 4f),

252 suggesting that both embryo-derived, and monocyte-derived $\mathrm{Tim}^{+} \mathrm{M} \phi$ require TAFA4

253 for survival pi. Consistently, the frequency of apoptotic Annexin ${ }^{+} \mathrm{Tim}^{+}$cells in UV-

254 exposed skin was higher for GINIP-DTR and TAFA4-KO mice than for control 255 littermates, whereas DN and MHC- $\mathrm{II}^{+}$subsets were unaffected (Extended data Fig.

256 8i-I). Thus, following exposure to UV, GINIP-DTR and TAFA4-KO mice present an 257 imbalanced $\mathrm{M} \phi$ response, with impaired survival of IL-10-producing $\operatorname{Tim}^{+} \mathrm{M} \phi$ and 258 larger numbers of Inflam $\mathrm{M} \phi$. TAFA4 deficiency therefore affects both the 259 maintenance of dermal $\mathrm{Tim} 4^{+} \mathrm{M} \phi$ and their IL-10 production capacity.

261 Intradermal injections of IL-10-blocking antibodies before UV irradiation in WT mice 262 induced a phenotype similar to that of Tafa4-KO mice (Extended data Fig. 9a-c), 263 suggesting that IL-10 upregulation is essential for $\operatorname{Tim} 4^{+} \mathrm{M} \phi$ maintenance. Similarly, 264 IL-10-deficient mice (Il-10 gfp/gfp mice) exposed to UV had smaller numbers of Tim4 ${ }^{+}$ $265 \mathrm{M} \phi$, larger numbers of Inflam $\mathrm{M} \phi$, and greater skin damage (Extended data Fig. 9d266 f). IL-10 defects are, therefore, sufficient to mimic the phenotype of GINIP-DTR and 267 Tafa4-KO mice, suggesting that IL-10 acts downstream from TAFA4 signaling.

268 Consistent with the crucial role of IL-10-producing $\mathrm{M} \phi$ in this model, we found that 269 resident M $\phi$ depletion by CSF-1R-blocking antibody (AFS98) ${ }^{17}$ treatment before UV 270 exposure decreased total skin IL-10 content (Extended data Fig. 9g-j). Monocyte 271 infiltration after resident $\mathrm{M} \phi$ depletion led to the rapid reconstitution of $\mathrm{MHC}-\mathrm{II}^{+}$and 
DN M $\phi$ subsets, whereas Tim $4^{+} \mathrm{M} \phi$ generation took up to six days (Extended data

273 Fig. 9k). M $\phi$-depleted mice presented increases in the number of Inflam $M \phi$ and skin

274 damage pi (Extended data Fig. 91-n), demonstrating the requirement of a

275 TAFA4/dermal resident $\mathrm{M} \phi$ axis for regulating Inflam $\mathrm{M} \phi$ responses. Furthermore,

276 adoptive transfer of sorted dermal $\mathrm{Tim}^{+} \mathrm{M} \phi$ in the skin of GINIP-DTR mice pi was

277 sufficient to limit tissue damage (Extended data Fig. 10a-d). Thus, the early loss of

278 dermal resident $\mathrm{Tim}^{+} \mathrm{M} \phi$ determines the tissue damage and repair defect rates in

279 GINIP-DTR and TAFA4-KO mice.

280 Finally, the phenotype of TAFA4-KO mice was rescued by intradermal injections of 281 recombinant TAFA4 pi (Fig. $\mathbf{4 g}, \mathbf{h})$. TAFA4 administration was also sufficient to 282 upregulate $\operatorname{Tim}^{+} \mathrm{M} \phi$ IL-10 production in TAFA4-KO mice (Extended data Fig. 10e283 f). These data suggest that TAFA4 production by C-LTMR nerve endings after UV 284 exposure is necessary and sufficient to downregulate inflammatory processes by 285 modulating dermal $\mathrm{M} \phi$ homeostasis and IL-10 production.

\section{Discussion}

288 This study reveals a crucial immunoregulatory role of non-peptidergic GINIP ${ }^{+}$ 289 cutaneous sensory neurons in tissue repair, through TAFA4 production, promoting 290 early and persistent IL-10 production by dermal macrophages. TAFA4 ensures the 291 survival of embryo-derived $\operatorname{Tim}^{+} \mathrm{M} \phi$ and sustains their production of large amounts 292 of IL-10. This neuro-immune pathway downregulates Inflam M $\phi$ expansion, thereby 293 reducing inflammatory cytokine levels, and promoting skin repair (Extended data Fig. 294 10g). These data are consistent with the metabolic reprograming capacity of IL-10 $0^{29}$ 295 and with previous reports of IL-10 deficiency leading to excessive collagen deposition 296 during wound healing ${ }^{30}$. The regulatory role of TAFA4 is not limited to $\operatorname{Tim} 4^{+} \mathrm{M} \phi$. 
297 TAFA4 can also modulate the inflammatory profile of other macrophage subsets in

298 vitro and in vivo, including other $\mathrm{CD} 206^{+}$dermal $\mathrm{M} \phi$ subsets and peritoneal $\mathrm{M} \phi$.

299 Recent studies of genetic or chemical ablations of sensory neuron subsets have revealed

300 a regulatory role of the somatosensory nervous system in cutaneous immune

301 responses ${ }^{3,5-9}$, which may be pro- or anti-inflammatory. The considerable functional

302 and phenotypic heterogeneity of somatosensory neurons ${ }^{31-33}$, and their selective or

303 combinatorial stimulation, may account for immunoregulatory differences between

304 pathological contexts. Previous studies have identified an important role of TRPV1 ${ }^{+}$

305 neurons and the neuropeptide CGRP in bacterial and fungal infections and models of

306 sterile and imiquimod-induced cutaneous inflammation ${ }^{3,5-7,9}$. Our study reveals a

307 crucial immunomodulatory role of TAFA4 production by C-LTMRs in a sunburn-like

308 model of skin damage. By contrast, no such effect was observed in a model of

309 imiquimod-induced skin inflammation (Extended data Fig. 10h). It will therefore be

310 important to determine whether this novel neuroimmune regulatory pathway also plays

311 a role in the skin in other pathological contexts. The properties of TAFA4 could be 312 exploited therapeutically to modulate inflammation in other inflammatory diseases or 313 in patients with wound healing defects. 


\section{References}

1 Wynn, T. A. \& Vannella, K. M. Macrophages in Tissue Repair, Regeneration, and Fibrosis. Immunity 44, 450-462, doi:10.1016/j.immuni.2016.02.015 (2016).

2 Merad, M. et al. Langerhans cells renew in the skin throughout life under steady-state conditions. Nat Immunol 3, 1135-1141, doi:10.1038/ni852 (2002).

3 La Russa, F. et al. Disruption of the Sensory System Affects Sterile Cutaneous Inflammation In Vivo. $J$ Invest Dermatol 139, 1936-1945 e1933, doi:10.1016/j.jid.2019.01.037 (2019).

4 Lopes, D. M. \& McMahon, S. B. Ultraviolet Radiation on the Skin: A Painful Experience? CNS Neurosci Ther 22, 118-126, doi:10.1111/cns.12444 (2016).

5 Chiu, I. M. et al. Bacteria activate sensory neurons that modulate pain and inflammation. Nature 501, 52-57, doi:10.1038/nature12479 (2013).

6 Riol-Blanco, L. et al. Nociceptive sensory neurons drive interleukin-23-mediated psoriasiform skin inflammation. Nature 510, 157-161, doi:10.1038/nature13199 (2014).

7 Kashem, S. W. et al. Nociceptive Sensory Fibers Drive Interleukin-23 Production from CD301b+ Dermal Dendritic Cells and Drive Protective Cutaneous Immunity. Immunity 43, 515-526, doi:10.1016/j.immuni.2015.08.016 (2015).

8 Pinho-Ribeiro, F. A. et al. Blocking Neuronal Signaling to Immune Cells Treats Streptococcal Invasive Infection. Cell 173, 1083-1097 e1022, doi:10.1016/j.cell.2018.04.006 (2018).

9 Cohen, J. A. et al. Cutaneous TRPV1(+) Neurons Trigger Protective Innate Type 17 Anticipatory Immunity. Cell 178, 919-932 e914, doi:10.1016/j.cell.2019.06.022 (2019).

10 Gaillard, S. et al. GINIP, a Galphai-interacting protein, functions as a key modulator of peripheral GABAB receptor-mediated analgesia. Neuron 84, 123-136, doi:10.1016/j.neuron.2014.08.056 (2014).

11 Basbaum, A. I., Bautista, D. M., Scherrer, G. \& Julius, D. Cellular and molecular mechanisms of pain. Cell 139, 267-284, doi:10.1016/j.cell.2009.09.028 (2009).

12 Abraira, V. E. \& Ginty, D. D. The sensory neurons of touch. Neuron 79, 618-639, doi:10.1016/j.neuron.2013.07.051 (2013).

13 Abrahamsen, B. et al. The cell and molecular basis of mechanical, cold, and inflammatory pain. Science 321, 702-705, doi:10.1126/science.1156916 (2008).

14 Braz, J. M. \& Basbaum, A. I. Differential ATF3 expression in dorsal root ganglion neurons reveals the profile of primary afferents engaged by diverse noxious chemical stimuli. Pain 150, 290-301, doi:10.1016/j.pain.2010.05.005 (2010).

15 Urien, L. et al. Genetic ablation of GINIP-expressing primary sensory neurons strongly impairs Formalin-evoked pain. Sci Rep 7, 43493, doi:10.1038/srep43493 (2017).

16 Gomez Perdiguero, E. et al. Tissue-resident macrophages originate from yolk-sacderived erythro-myeloid progenitors. Nature 518, 547-551, doi:10.1038/nature13989 (2015).

17 Hoeffel, G. et al. C-Myb(+) erythro-myeloid progenitor-derived fetal monocytes give rise to adult tissue-resident macrophages. Immunity 42, 665-678, doi:10.1016/j.immuni.2015.03.011 (2015).

18 Hoeffel, G. \& Ginhoux, F. Fetal monocytes and the origins of tissue-resident macrophages. Cell Immunol 330, 5-15, doi:10.1016/j.cellimm.2018.01.001 (2018).

19 Ginhoux, F. \& Jung, S. Monocytes and macrophages: developmental pathways and tissue homeostasis. Nat Rev Immunol 14, 392-404, doi:10.1038/nri3671 (2014).

20 Guilliams, M., Mildner, A. \& Yona, S. Developmental and Functional Heterogeneity of Monocytes. Immunity 49, 595-613, doi:10.1016/j.immuni.2018.10.005 (2018).

$21 \mathrm{Li}$, L. et al. The functional organization of cutaneous low-threshold mechanosensory neurons. Cell 147, 1615-1627, doi:10.1016/j.cell.2011.11.027 (2011). 
22 Delfini, M. C. et al. TAFA4, a chemokine-like protein, modulates injury-induced

23 Reynders, A. et al. Transcriptional Profiling of Cutaneous MRGPRD Free Nerve mechanical and chemical pain hypersensitivity in mice. Cell Rep 5, 378-388, doi:10.1016/j.celrep.2013.09.013 (2013).

24 Tom Tang, Y. et al. TAFA: a novel secreted family with conserved cysteine residues and restricted expression in the brain. Genomics 83, 727-734, doi:10.1016/j.ygeno.2003.10.006 (2004).

25 Wang, W. et al. FAM19A4 is a novel cytokine ligand of formyl peptide receptor 1 (FPR1) and is able to promote the migration and phagocytosis of macrophages. Cell Mol Immunol 12, 615-624, doi:10.1038/cmi.2014.61 (2015).

26 Grimbaldeston, M. A., Nakae, S., Kalesnikoff, J., Tsai, M. \& Galli, S. J. Mast cellderived interleukin 10 limits skin pathology in contact dermatitis and chronic irradiation with ultraviolet B. Nat Immunol 8, 1095-1104, doi:10.1038/ni1503 (2007).

27 Yona, S. et al. Fate mapping reveals origins and dynamics of monocytes and tissue macrophages under homeostasis. Immunity 38, 79-91, doi:10.1016/j.immuni.2012.12.001 (2013).

28 Hoeffel, G. et al. Adult Langerhans cells derive predominantly from embryonic fetal liver monocytes with a minor contribution of yolk sac-derived macrophages. $J \operatorname{Exp}$ Med 209, 1167-1181, doi:10.1084/jem.20120340 (2012).

29 Ip, W. K. E., Hoshi, N., Shouval, D. S., Snapper, S. \& Medzhitov, R. Antiinflammatory effect of IL-10 mediated by metabolic reprogramming of macrophages. Science 356, 513-519, doi:10.1126/science.aal3535 (2017).

30 Eming, S. A. et al. Accelerated wound closure in mice deficient for interleukin-10. Am $J$ Pathol 170, 188-202, doi:10.2353/ajpath.2007.060370 (2007).

31 Cavanaugh, D. J. et al. Distinct subsets of unmyelinated primary sensory fibers mediate behavioral responses to noxious thermal and mechanical stimuli. Proc Natl Acad Sci U $S$ A 106, 9075-9080, doi:10.1073/pnas.0901507106 (2009).

32 Usoskin, D. et al. Unbiased classification of sensory neuron types by large-scale singlecell RNA sequencing. Nat Neurosci 18, 145-153, doi:10.1038/nn.3881 (2015).

33 Sharma, N. et al. The emergence of transcriptional identity in somatosensory neurons. Nature 577, 392-398, doi:10.1038/s41586-019-1900-1 (2020). 
412 Figure 1: GINIP ${ }^{+}$sensory neurons prevent fibrosis after UV exposure

413 a, Experimental scheme indicating the phases of skin inflammation, resolution and 414 repair after overexposure to UV radiation (See also Extended data fig. 1a for 415 inflammatory and pro-repair gene expression pi). b, Atf3 mRNA levels in C2/C3 DRGs over time in UV-irradiated (purple dots) and non-exposed mice (gray dots), ( $n=5$ mice 417 per group). c, Confocal images of GINIP (red), CGRP (blue) and ATF3 (green) 418 expression in DRGs, from unexposed (No UV) and UV-exposed mice (D3 UV). (See 419 also C2/C3 DRG characterization in Extended data fig. 1b-f). d, Percentage of ATF3 ${ }^{+}$ 420 neurons from C2/C3 DRGs positive for CGRP (blue) or GINIP (red) at D3 pi ( $\mathrm{n}=8$ mice 421 per group). e, Changes in ear thickness over time pi, in control DTR (blue) and GINIP422 DTR mice (red), ( $n=7$ mice per group). f, Representative control DTR (left) or GINIP423 DTR mice (right) ears on D14 pi. (see also neuron depletion in GINIP-DTR skin, 424 Extended data fig. 2a-b). Representative H\&E staining of ear sections from control 425 DTR (g), and GINIP-DTR mice (h), at D35 pi. Fibrosis (i), and cumulative 426 histopathological score (j), at D35 pi ( $n=5$ mice per group). (See also Extended data 427 fig. 2d-j for detailed analysis of histopathological score, scratching behavior and back 428 skin analysis). Data are presented as mean \pm SEM and representative for at least two 429 independent experiments. One-way ANOVA with Tukey tests, except for $\mathbf{b}$ and $\mathbf{e}$, two430 way ANOVA and d, Student's $t$-test.

Figure 2: Dermal-resident Tim4 $4^{+}$macrophage maintenance is compromised in UV-exposed GINIP-DTR mice

434 a, Confocal analysis of ear skin from Nav1.8-RFP mice stained for GINIP ${ }^{+}$neurons 435 (green) and CD206 ${ }^{+}$dermal resident $\mathrm{M} \phi$ (blue). b, Distance $(\mu \mathrm{m})$ between $\mathrm{CD}^{2} 26^{+} \mathrm{M} \phi$ 
and GINIP $^{+}$axons $(\mathrm{n}=97 \mathrm{M} \phi)$. (See also Extended data Fig. 3g for higher

437 magnification). c, Cluster analysis and t-SNE distribution of $\mathrm{Mo} / \mathrm{M} \phi$ subsets.

438 Monocyte-related subsets $\left(\mathrm{CCR}^{+}\right)$clustered as $\mathrm{Ly}^{+} \mathrm{C}^{+} \mathrm{Mo}\left(\mathrm{Ly} \mathrm{C}^{+}\right.$, orange), Int. Mo 439 (Ly6C $^{\text {int }}, \mathrm{MHCII}^{\text {int }}$, light green), PMo (Ly6C-${ }^{-}, \mathrm{MHCII}^{-}, \mathrm{CD}^{-}$, dark green) and their 440 derivatives MoDC (Ly6C ${ }^{-}, \mathrm{MHCII}^{+}$, blue) and Inflam M $\phi\left(\mathrm{Ly}^{-}, \mathrm{MHCII}^{-}, \mathrm{CD}^{+}\right.$, $441 \mathrm{CD}^{206}$, pink). Dermal resident $\mathrm{M} \phi\left(\mathrm{CD}^{-} 4^{+} \mathrm{CD} 206^{+}\right)$subsets were identified as $442 \mathrm{MHCII}^{+} \mathrm{M} \phi\left(\mathrm{MHCII}^{+}, \mathrm{Tim}^{-}\right.$, red $), \mathrm{DN} \mathrm{M} \phi\left(\mathrm{MHCII}^{-}, \mathrm{Tim}^{-}\right.$, purple $)$and $\mathrm{Tim}^{+} \mathrm{M} \phi$ 443 (MHCII-, Tim4 $^{+}$, cyan). (See also Extended data Fig. 3a, and 4a-c; 4e-g for detailed 444 gating strategy and t-SNE analysis, and Extended data Fig. 4d-f for subset 445 quantification). $\mathbf{d}$, $\mathrm{t}-\mathrm{SNE}$ clustering of $\mathrm{Mo} / \mathrm{M} \phi$ subsets in DTR (left) or GINIP-DTR 446 (right) mice at D14 pi. e, Absolute numbers of $\mathrm{Tim}^{+} \mathrm{M} \phi$ (cyan) or $\mathbf{f}$, Inflam $447 \mathrm{M} \phi$ (pink), per mg ear skin ( $n=10-14$ mice per group) from DTR (gray) or GINIP-DTR 448 (colored) mice, between days 0 and 14 pi. Data are presented as mean \pm SEM and 449 representative for at least two independent experiments. One-way ANOVA with Tukey 450 tests (ns: non-significant).

Figure 3: TAFA4, a neuropeptide expressed by C-LTMRs, regulates IL-10 453 production by macrophages and prevents $\mathrm{UV}$-induced skin fibrosis

454 a, Representative confocal images of C3 DRGs from WT mice on day 3 (D3) pi stained 455 with IB4 (blue), anti-TAFA4 (red) and anti-ATF3 antibodies (green). b, Percentage $456 \mathrm{ATF}^{+}$neurons positive for IB4 (blue) and TAFA4 (red) ( $n=8$ mice per group). c, 457 Quantification of TAFA4 protein (ELISA) in WT mice ear skin from D0 to D35 pi 458 ( $n=6-18$ mice per group). (See Extended data Fig. 5 for C-LTMRs analysis and 459 TAFA4 detection). d, Representative Picro Sirius staining of ear sections from WT 460 (top) and Tafa4-KO (bottom) mice at D35 pi and e, collagen deposition: percentage 
461 fibrosis (yellow pixels) over the total area (red + yellow pixels), ( $n=9$ mice per group).

462 f, TNF $\alpha$ and g, IL-10 protein levels (CBA) in ear skin lysates ( $n=4-8$ mice per group).

$463 \mathbf{h}$, Relative $I l 10 \mathrm{mRNA}$ levels in sorted dermal CD206 ${ }^{+} \mathrm{M} \phi$ from WT (blue) and Tafa4-

$464 \mathrm{KO}$ (red) mice between days 0 and 10 pi (pooled from 3 independent sortings, each

465 with $n=3$ mice per group). i, Relative $T n f a, I l 1 b$ and $I l 10$ mRNA levels (RT-qPCR) for

466 purified peritoneal $\mathrm{M} \phi($ ThioM $\phi)$ activated in vitro with LPS (100 ng/ml) without (blue)

467 or with TAFA4 (100 nM; green), ( $n=5-8$ independent samples). Data are presented as

468 means \pm SEM and representative for at least two independent experiments. One-way

469 ANOVA with Tukey tests, except for $\mathbf{b}$ and $\mathbf{f}$, Student's $t$-test $\mathbf{c}$, Kruskal-Wallis test,

470 and d, two-way ANOVA (ns: non-significant; ${ }^{*} p<0.05 ; * * p<0.01 ; * * * p<0.001$ ). 
Figure 4: The TAFA4-IL-10 axis promotes $\mathrm{Tim}^{+}{ }^{+}$macrophage survival and tissue repair functions

a, Experimental strategy for generating CD45.1 BM chimeras in CD45.2 WT or Tafa4KO recipient mice and monitoring monocyte-derived cells pi. b, FACS and t-SNE analysis of skin Mo/M $\phi$ subsets 7 days pi, as described in Fig. 2 (See also Extended data fig. 8f-h for detailed BM chimera analysis). c, t-SNE analysis of CD45.2 $2^{+}$and CD45. $1^{+}$clusters (yellow and black) and intracellular staining for IL-1 $\beta$, TNF $\alpha$ and IL10 (red). d, Absolute numbers of Inflam M $\phi$ in WT control (gray) and Tafa4-KO BMchimeric mice (pink). e, Absolute numbers of CD45.2 $2^{+} \mathrm{Tim} 4^{+} \mathrm{M} \phi$ (left) and percentage of $\mathrm{CD} 45.1$ chimerism in total $\mathrm{Tim}^{+} \mathrm{M} \phi$ (right) from WT (gray) and Tafa4-KO BMchimeric mice (cyan). f, Absolute numbers of total (CD45.1 $1^{+}$and CD45.2 $\left.{ }^{+}\right) \mathrm{Tim}^{+} \mathrm{M} \phi$ in WT (gray) and Tafa4-KO BM-chimeric mice (cyan), ( $n=6-10$ mice per group). $\mathbf{g}$, Intradermal TAFA4 protein injection in Tafa4-KO mice (green arrows) and changes in ear thickness over 21 days pi in saline-treated WT (blue) and Tafa4-KO (red) mice, or Tafa4-KO mice treated with TAFA4 (green) ( $n=14$ mice per group). h, Absolute numbers of Inflam $\mathrm{M} \phi$ (left) and $\mathrm{Tim} 4^{+} \mathrm{M} \phi$ (right) in ear skin on day 14 pi ( $n=11$ mice per group). Data are presented as mean \pm SEM and representative for at least two independent experiments. One-way ANOVA with Tukey tests except for (g), two-way ANOVA (ns: non-significant; ${ }^{*} p<0.05 ; * * p<0.01 ; * * * p<0.001$ ).

\section{Methods}

$494 \quad \underline{\text { Mice }}$

495 C57/Bl6J mice were bought from Janvier Labs (https://www.janvier-labs.com). 496 GINIP-DTR mice were obtained by crossing GINIPflx/+ line ${ }^{10}$ with mice expressing 497 the CRE recombinase from Nav1.8 locus (Nav1.8 $\mathrm{Cre} / \mathrm{Cre}$ mice) ${ }^{13}$. One single 
diphtheria toxin (DT; $10 \mu \mathrm{g} / \mathrm{kg}$ ) injection in Nav1.8 $\mathrm{Cre/+}$ GINIPflx/+ (hereafter called GINIP-DTR mice) allowed the specific ablation of GINIP ${ }^{+}$sensory neurons ${ }^{15}$. Nav1.8 +/+ GINIP $f\left(x /+^{+}\right.$littermates (hereafter called DTR mice) treated with DT were

501 used as controls throughout. Nav1.8 ${ }^{\mathrm{rfp}}$ were obtained by crossing Nav1.8 $\mathrm{Cre/+}$ with flxR26-RFP mice. Homozygotes $I L-10 g p f / g f p$ mice, described previously ${ }^{34,35}$ were used with their heterozygotes IL-10gfp/wt control littermates. Tafa4-KO mice were generated in Aziz Moqrich's lab (IBDM, AMU, France) and described previously ${ }^{22}$. CX3CR1 CreERT2:R26-YFP mice were described previously ${ }^{27}$. All the mice used were

506 bred and maintained under specific pathogen-free conditions at the Centre d'Immunophénomique (Ciphe) de Marseille and at the Centre d'Immunologie de Marseille Luminy (CIML). Mice were housed under a standard 12 h:12 h light-dark cycle with ad libitum access to food and water. Age-matched (6-12 weeks old, unless specified in figure legend) and sex-matched littermate mice were used as

511 controls. All experiments were conducted in accordance with institutional committee recommendations (Comité d'Éthique de Marseille no. 14-APAFiS \# 18469-2019011417196625v2) and French and European guidelines for animal

514 care.

Bone marrow chimera generation

517 Age and sex matched WT or Tafa4-KO mice (CD45.2+) were anaesthetized with 518 ketamine/xylazine $(10 \mu \mathrm{l} / \mathrm{g}, 2 \%$ Imalgene500/ 5\% Rompun). Mice were then 519 irradiated with 6,5Gy from an $\gamma$-ray irradiator. A lead shield was used to protect 520 the ear's skin from $\gamma$-irradiation. Six hours after irradiation, 30mg/kg Busulfan 521 (Sigma) ${ }^{36}$ was injected (i.p.) to deplete remaining myeloid progenitors in recipient 522 mice. Twelve hours later the mice were reconstituted with 5 million CD 45.1+ bone 523 marrow (BM) cells. Mice were treated with Bactrim (in drinking water) for two 524 weeks for a total recovery time of 6 weeks before analyzing the blood CD45.1 525 chimerism and were then used for experiments.

\section{$527 \quad$ Lineage tracing study}

528 CX3CR1 ${ }^{\text {Creert2:R26R-EYFP+/+ }}$ were used as described ${ }^{27}$. Tamoxifen (2mg Tam/mice; $52940 \mathrm{mg} / \mathrm{ml}$ in corn oil; Sigma) was injected i.p. to pregnant mice at E16.5 days postconception (dpc). Day of embryonic development (E) was estimated by taking the 
531 day of vaginal plug observation as E0.5 dpc. Caesarian sections were carried out 532 at term and neonates were fostered by lactating females. In this model, Tam-

533 induced recombination leads to irreversible expression of the enhanced yellow

534 fluorescent protein (EYFP) in CX3CR1+embryonic precursors and their progeny

535 (i.e. fetal monocytes and differentiated $\mathrm{M} \phi$ at the time of Tam injection; for review 536 see $\left.^{37}\right)$.

\section{$\underline{\text { UV-induced skin injury }}$}

539 Mice were anaesthetized with ketamine/xylazine, shaved and depilated (Veet 540 Cream) and then left untreated or exposed to UV ( $\lambda 254 \mathrm{~nm}$; voltage: $8 \mathrm{~W}$; source:

$54130 \mathrm{~cm}$ from the target) for $25 \mathrm{~min}$. The protocol described previously ${ }^{2}$ was adapted 542 so that the ear skin of WT mice can recover in 35 days after UV exposure. Ear 543 thickness was measured every other day with a caliper (under isoflurane 544 anesthesia) and compared to the ear thickness of control untreated mice.

\section{$546 \quad$ Histology and histopathological analysis}

547 At different time pi, mice were lethally anesthetized ketamine/xylazine $(20 \mu \mathrm{l} / \mathrm{g})$ 548 and perfused with $10 \mathrm{ml}$ of PBS. Ears were then collected and fixed with formol 549 (for at least $1 \mathrm{~h}$ ), then cryoprotected with sucrose $30 \%$ overnight and embedded 550 in paraffin. Skin sections of $5 \mu \mathrm{m}$ were stained with hematoxylin and eosin, 551 Masson's trichrome or picrosirius red. Histological scores were blindly assessed 552 by a pathologist following the criteria described below. Leukocyte infiltration, 553 epidermal thickness and fibrosis were evaluated and a score was assigned based 554 on severity for each criterion. Fibrosis was also evaluated using a semi555 quantitative binary score based on percentage of surface modified by fibrotic 556 changes. A cumulative score was then obtained.

$558 \quad$ Histopathological grading

\section{Inflammation/leukocyte infiltration}

560 (0): No inflammation; (1): Mild dermatitis (less than 10 leukocytes per High 561 Power Fields, HPF); (2): Moderate dermatitis (less than 50 leukocytes/HPF); (3): 562 Marked dermatitis (less than 100 leukocytes/HPF); (4): Severe dermatitis (more 563 than 100 leukocytes/HPF). 
564 Epidermal thickness

565 (0): Normal thickness; (1): Mild hyperplasia (less than 4 cellular layers); (2):

566 Moderate hyperplasia (more than 4 cellular layers); (3): Marked hyperplasia

567 (markedly thickened, stratification and epidermal crest)

568 Wound healing/fibrosis

569 (0): No granulation tissue, no fibrosis; (1): Early granulation tissue and mild 570 fibrosis; (2): Moderate fibrosis (hair follicle obliteration); (3): Marked fibrosis

571 (persistent granulation tissue/cartilage obliteration)

\section{Fibrosis extension}

573 (1): Focal or multifocal fibrosis (less than 50\% fibrosis per HPF); (2): Extensive

574 fibrosis (more than $50 \%$ fibrosis per HPF)

575

576 Dorsal Root Ganglia (DRG) tissue sections

577 After lethal anesthesia and perfusion with $5 \mathrm{ml}$ of PBS and $25 \mathrm{ml}$ of $4 \%$ 578 paraformaldehyde (PFA), C2/C3 DRG were carefully dissected from the spinal 579 cord under a binocular lens, fixed for 1 hour with 4\% PFA and then cryoprotected 580 with $30 \%(\mathrm{w} / \mathrm{v})$ sucrose overnight before being included in OCT (Tissue-Tek, 581 Sakura) and stored at $-80^{\circ} \mathrm{C}$. DRG samples were sectioned at $10 \mu \mathrm{m}$ using a 582 standard cryostat (Leica).

583

584 In situ Hybridization on $D R G$

585 In situ hybridization was carried out following the protocol described in ${ }^{15}$. Briefly, 586 RNA probes were synthesized using gene-specific PCR primers and cDNA 587 templates from mouse DRG. The following oligonucleotides were used for the 588 nested PCRs for probe synthesis:

589 Tafa4-F1: TGCTCAGAAGTTCATAGCCAAA

590 Tafa4-R1: TAAAGGAACATTTGCAAGCTCA

591 Tafa4-F2: ATATGTGCAGTGTGG

592 Tafa4-R2 + T7: TAATACGACTCACTATAGGGCAGCCAAGTTCAAAC

593

594 DRG sections were treated with proteinase $\mathrm{K}$, triethanolamine and acetic 595 anhydride solutions. Digoxigenin labeled probes were hybridized overnight at $59655^{\circ} \mathrm{C}$ after $2 \mathrm{~h}$ of prehybridization. The slides were treated with $0.2 \mathrm{X}$ SSC baths 
then blocked at room temperature with $10 \%$ Goat serum and incubated with antidigoxigenin antibodies (Roche). Final detection was achieved using Cyanine 3 TSA plus kit (Perkin Elmer).

600

Immunofluorescent and confocal analysis of DRG neuron activation

602 We used the expression of ATF3 (Activating Transcription Factor 3) as a proxy for 603 neuronal injury and activation ${ }^{38}$. DRG sections were saturated and permeabilized 604 with a PBS 1X, 3\% BSA, 0,3\% Triton 100X and 10\% Donkey serum (Sigma; 605 \#D9663-10ML) solution for 1 hour at room temperature (RT) before adding the 606 primary anti-mouse TAFA4 antibody (rat clone 1D8, 1:1000), the anti-mouse 607 GINIP (rat, 1:1000, Aziz Moqrich's lab, IBDM) or the anti-mouse GINIP (rabbit, 608 1:1000, Invitrogen \#PA5-71131), the anti-mouse ATF3 (rabbit, 1:200, Santa Cruz 609 \#SC-188 or Abcam \#ab207434), the anti-TH (1:300, Merck Millipore, \#AB152) 610 and anti-mouse CGRP (goat, 1:1000, Abcam \#ab36001) for 24 hours at $4^{\circ} \mathrm{C}$. After 611 several washes, donkey secondary antibodies (anti-mouse, \#715-585-151; anti612 rat, \#712-545-153; anti-rabbit, \#711-585-152; and anti-goat, \#705-605-147; 613 from Jackson ImmunoResearch, 1:500) were added for 45 minutes at room 614 temperature. The staining with the lectin isolectin B4 (IB4) coupled to AlexaFluor 615647 (AF647; 1:200, Invitrogen) was performed in TNT buffer (Tris HCl 100 nM, $616 \mathrm{NaCl} 150 \mathrm{nM}$, Tween-20 0,1\% pH 7,5) for 45 minutes at room temperature. Images 617 were acquired using a confocal microscope LSM780 (Zeiss) and analyzed using 618 ZEN 2.3 and ImageJ Software.

619

Skin cell isolation

621 After lethal anesthesia and intracardiac perfusion with $5 \mathrm{ml}$ of PBS, ears were 622 collected and dorsal and ventral layer separated, finely minced with scissor in 623 complete medium (RPMI+L-Glutamine, 10\% FCS) and incubated $1 \mathrm{~h}$ at $37^{\circ} \mathrm{C}$ with $6241 \mathrm{mg} / \mathrm{ml}$ DNase (Roche) $0.2 \mathrm{mg} / \mathrm{ml}$ Dispase (GIBCO) and $0.2 \mathrm{mg} / \mathrm{ml}$ collagenase 625 type IV (Sigma; \#C5138-500MG). Tissues were then dissociated using $2.5 \mathrm{ml}$ 626 syringes and 18G needles and filtered on cell strainer (100 $\mu \mathrm{m}, \mathrm{BD})$, washed in 627 FACS buffer (PBS-2mM EDTA, FCS 2\%) to obtain a homogeneous cell suspension 628 ready for staining. 
631 After lethal anesthesia and intracardiac perfusion with $5 \mathrm{ml}$ of HBSS $10 \mathrm{X}\left(\mathrm{Mg}^{-} \mathrm{Ca}^{2-}\right.$

632 ), HEPES $5 \mathrm{mM}$, D-Glucose $12.5 \mathrm{mM}$, and penicillin/streptomycin (P/S) 1\% 633 solution, DRGs were carefully extracted and digested twice with a $0.2 \mathrm{mg} / \mathrm{ml}$ 634 collagenase type II (Gibco), and $0.5 \mathrm{mg} / \mathrm{ml}$ dispase (Gibco) solution for 30 minutes 635 at $37^{\circ} \mathrm{C}$. After several wash with Neurobasal complete medium (B-27 2\%, L636 glutamine $20 \mathrm{mM}, \mathrm{P} / \mathrm{S}$ 1\%), DRGs were mechanically dissociated using 3 needles 637 of decreasing diameter (18G, 22G, 26G). After a filtration on a cell strainer $(70 \mu \mathrm{m}$, 638 Miltenyi Biotec), a Percoll gradient was used with $12.8 \%$ and $28 \%$ density in 639 Leibovitz-15 complete medium (FCS 5\% and P/S 1\%) to eliminate cell debris. 640 After centrifugation and several washes, the cell suspension was ready for 641 staining.

642

643 Antibodies for flow cytometry

644 We adapted our FACS gating strategy from previous studies ${ }^{39}$, adding EpCAM, 645 Langerin (CD207) or CD206 and Tim4 to decipher the Mo/M $\phi$ compartment of the 646 skin. Antibodies were purchased from BioLegend or BD Biosciences: CD45-BV785 647 1:500 (30F11), CD45.1-BV605 1:200 (A20), CD11b-BV510 1:1000 (M1/70), 648 CD64-BV711 1:300 (X54-5/7.1), Ly6C-421 1:300 (AL-21), F4/80-PECF594 1:400 649 (T45-2342), CD11c-BUV395 1:200 (N418), EpCAM-AF594 1:500 (G8.8), CD207650 APC (929F3.01; Dendritics, \#DDX0362), CD206-APC 1:300 (C068C2), Tim4651 PeCy7 (RMT4-54) Ly6G-APC-Cy7 1:300 (1A8), IA-IE A700 1:500 (M5/114.15.2), 652 CD24-BUV737 1:500 (M1/69). Mast cells were characterized as previously 653 described40,41, CD117-BV605 (c-kit; 2B8; 1:300), CD103-PerCP-Cy5.5 (2E7; 654 1:500), anti-TNFo-PE 1:100 (MP6-XT22) anti-IL-10-PE 1:100 (JES5-16E3); CCR2655 PE 1:200 (475301; R\&D System), IL-1 $\beta$-PE Ab 1:100 (NJTEN3; eBioscience), anti656 CD4 PE-Cy7 (RM4-5; BD), anti-TCR $\alpha \beta$ APC-Cy7 (H57-597; BD), anti-FoxP3-A488 657 1:200 (Clone FJK-16s; eBioscience), anti-DTR 1:300 (anti-hHB-EGF; AF-259-NA; 658 R\&D System), anti-GFP (Invitrogen, \#A-21311).

660 Flow cytometry

661 Cell suspensions were plated in 96 well $\mathrm{U}$ bottom plates and stained at $4^{\circ} \mathrm{C}$. Cells 662 were incubated $40 \mathrm{~min}$ at $4{ }^{\circ} \mathrm{C}$ in FACS buffer with antibodies and anti-Fc receptor 
663 blocking antibody (clone: 2.4 G2). Cells were then washed and fixed (fixation 664 buffer, from FoxP3 kit; eBioscience; \#00-5521-00) until analysis. For intra-cellular 665 cytokine staining, cell suspensions were permeabilized (Fix/perm, from FoxP3 666 kit; eBioscience) and stained with anti-cytokine antibodies during $1 \mathrm{~h}$ for IL-1 $\beta$ and 667 TNF $\alpha$ or overnight for IL-10. For IL-10 expression analysis in FoxP3+ Treg cells, 668 total skin homogenates were incubated during $6 \mathrm{~h}$ in complete medium 669 supplemented with PMA (200ng/ml; Sigma) and ionomycin (1mg/ml; Sigma). 670 During the last 4h, Golgi Plug (1:1000; BD Biosciences; \#554724) was added. Cell 671 suspensions were then washed extensively at $4^{\circ} \mathrm{C}$, surface stained, fixed and then 672 processed for intracellular staining. Dead cells were excluded from the analysis by 673 counter-gating using the live/dead fixable blue Dead Cell Stain kit UV (L23105; 674 Invitrogen). Multiparameter FACS analysis was performed using an LSR X20 675 system (BD). Absolute numbers for each population were obtained using Quanti 676 Beads (BD Biosciences, \#556296). Data analysis was performed using Flowjo 677 software (Tree Star, Inc.).

\section{t-distributed Stochastic Neighbor Embedding (t-SNE)}

680 t-SNE analyses were performed with FlowJo ${ }^{\mathrm{TM}}$ version 10 software (FlowJo LLC). 681 We used an equal number of mice for each genotype or experimental condition 682 considered. In each group, the same number of cells was used to allow an 683 equivalent contribution of each individual mice in the analysis. This equal cell 684 number was selected using the DownSample plugin. Then, cells from each mouse 685 were merged using the concatenate tool and barcoded to track and distinguish 686 them. Finally, t-SNE analyses were performed using different markers, indicated 687 in the figure legends. The color code representing the expression level of each 688 marker in the heatmaps (depicted in extended data figures), is normalized on the 689 median intensity value for a given marker. Data are represented using a four-color 690 scale with blue, green-yellow and red indicating respectively low intermediate 691 and high expression levels.

693 Immunofluorescent staining and confocal microscopy analysis of ear skin

694 After lethal anesthesia and perfusion with 10ml PBS, ears from DT-treated GINIP695 DTR and -DTR control mice were collected and ear dorsal and ventral layers were 
separated, fixed for 1 hour with 4\% PFA, washed, permeabilized and saturated with a PBS, $3 \%$ BSA, $0,2 \%$ Triton $100 \mathrm{X}$ and $10 \%$ donkey serum and then stained with the primary anti-mouse GINIP antibody (rat, 1:1000, Aziz Moqrich's lab, IBDM) , the anti-TH antibody (rabbit,1:300; Merck Millipore, \#AB152) overnight at $4^{\circ} \mathrm{C}$. After several washes, a donkey secondary anti-rat-488 and anti-rabbitAF594 antibody (Jackson Immunoresearch, 1:500) were added $1 \mathrm{~h}$ at $4^{\circ} \mathrm{C}$, washed, and then the anti-Beta III Tubulin-AF647 antibody (1:300 TUJI; Biolegend, \#BLE801210) or the anti-CD206-APC 1:300 (rat, 1:300; clone C068C2, Biolegend, \#BLE141708), were added for additional 45 minutes at room temperature. After several washes slices were then mounted on coverslips with mounting medium and image acquisition was performed on Confocal LSM780 (Zeiss) and analyzed with ZEN and ImageJ software.

\section{Bone marrow-derived macrophage (BMDM) generation}

710 Femora bones were isolated from WT or FPR1-KO mice (We thank Pr Monica 711 Lucattelli and Pr Laurence Zitvogel for providing BM cells from FPR1-KO mice), 712 cut in half and placed in $0.5 \mathrm{ml}$ Eppendorf tubes pre-perforated on their bottom 713 part. The small tubes with two $1 / 2$ bone were placed within $1.5 \mathrm{ml}$ Eppendorf tubes. 714 The tubes were then centrifuged 10sec at 10000rpm and BM cell pellets were 715 resuspended in red blood cell lysis buffer for 3 minutes and washed in PBS. BM 716 cells were then resuspended at $10^{6} / \mathrm{ml}$ in complete DMEM (DMEM, SVF 10\%, P/S, 717 1\% L-Glu) supplemented with 20\% conditioned L929 media during 5 days to 718 generate bone marrow derived-macrophages (BMDM) and used as ThioM $\phi$ for in 719 vitro experiments.

Thioglycollate-elicited macrophage (ThioM $\phi)$ generation and in vitro treatment

722 Peritoneal macrophages were isolated from WT mice by peritoneal wash three 723 days after 3\% Thioglycollate (Sigma) i.p. injection. Peritoneal washes were then 724 stained with biotin-associated lineage cocktail antibodies (CD3, CD19, NK1.1, 725 Ly6G and Siglec F), counterstained with anti-biotin microbeads (Miltenyi Biotec) 726 and then purified using magnetic LS columns. Peritoneal macrophage 727 preparations were then cultured in complete DMEM (DMEM, SVF 10\%, P/S, 1\% LGlu) at $10^{6}$ ThioM $\phi / \mathrm{ml}$ in a 12 -well plates (BD) with or without LPS (100ng.ml, 
729 Sigma) in the presence of not of 100nM TAFA4. After 16 hours, macrophages were 730 lysed using RLT medium (Qiagen) then pass through a QIAShredder column 731 (Qiagen) and store at $-80^{\circ} \mathrm{C}$.

$733 \quad$ Migration assay

734 BMDM from WT or FPR1-KO mice were incubated on the top chambers of 735 transwell plates (Costar) in complete DMEM, while the bottom chambers of the 736 transwell plates were filled with complete DMEM for negative control, or complete 737 DMEM containing CCL2 (MCP-1; 10nM) for positive control of migration, or 738 increasing concentrations of TAFA4 ( 1 to $1000 \mathrm{nM}$ ), during $8 \mathrm{~h}$ at $37^{\circ} \mathrm{C}$. The bottom chambers were then analyzed by FACS for macrophages content.

Dermal resident macrophage depletion.

742 WT or Tafa4-KO mice were injected two consecutive days with $500 \mu \mathrm{g}$ of the CSF743 1R blocking antibody (clone AFS98; InVivoMAb, BioXcell \#BE0213) or the isotype 744 control (IgG2a; clone 2A3; InVivoMAb, BioXcell \#BE0089). One day after the last 745 injection, mice tissues were collected and analyzed for Macrophage depletion by 746 flow cytometry.

Dermal resident macrophage isolation

749 Ears skin from WT or Tafa4-KO mice were dissociated and stained as previously 750 described, and CD206+ dermal resident macrophages were cell sorted on a FACS 751 Aria III following the Figure 2 gating strategy as livedeadneg, CD45+ ${ }^{+} C^{\text {neg }}$ 752 CD11b+CD24-Ly6G-CCR2-Ly6C-CD64 ${ }^{+}$CD206 ${ }^{+}$, then lysed using RLT medium 753 (Qiagen) then pass through a QIAShredder column (Qiagen) and store at $-80^{\circ} \mathrm{C}$. 754 Alternatively, $\mathrm{Tim} 4^{+} \mathrm{M} \phi$ or Inflam $\mathrm{M} \phi\left(\mathrm{CD}^{+} 4^{+} \mathrm{CD} 206^{-}\right)$were sorted as presented in 755 Extended data Figure 10b.

Intradermal adoptive transfer of Inflam and Tim $4^{+}$macrophages.

758 DTR and GINIP-DTR mice were exposed to UV and then intradermally injected at 759 D1 pi with $2000 \mathrm{M} \phi / 10 \mu \mathrm{l}$ PBS per ears.

761 Imiquimod-induced cutaneous damage. 
762 Mice (WT or Tafa4-KO mice) were daily treated with $5 \mathrm{mg}$ of 5\% imiquimod cream

763 (IMQ) applied topically to dorsal and ventral aspects of ear skin as describe6,

764 during six days and evolution of the ear thickness $(\mu \mathrm{m})$ was followed every day

765 with a caliper.

766

767

TAFA4 rescue and in vivo blocking of $I L-10$

768 For TAFA4 rescue experiments, $20 \mu \mathrm{l}$ of recombinant TAFA4 $(25 \mu \mathrm{g} / \mathrm{ml})$ or saline

769 solution only, were intradermally injected every other day following the UV-

770 treatment until day 8 pi in each ear of Tafa4-KO or control mice as indicated in

771 figures.

772 For IL-10 blocking experiments, $20 \mu \mathrm{l}$ of anti-IL-10 blocking antibody $(0.5 \mathrm{mg} / \mathrm{ml}$;

773 R\&D, \#MAB417) or the isotype control (rat IgG1; clone JES052A5) were

774 intradermally injected every other day following the UV-treatment until day 8 pi

775 in each ear of Tafa4-KO or control mice as indicated in figures.

776

$777 \quad$ Gene expression analysis

778 Total RNA was isolated from mouse ears, exposed or not to UV irradiation or from

779 BMDM or ThioM $\phi$ activated with or without LPS and TAFA4. Ears were

780 dilacerated by FastPrep-24 (MpBio) in lysis matrix A tubes (MpBio). RNA was

781 isolated using a fibrous RNeasy minikit (QIAGEN). Reverse transcription was

782 performed using Superscript RTII (Invitrogen). Preamplification was performed

783 using specific Taqman probes (Applied Biosystems) for each targeted gene using

784 the pre-amplification master mix (Fluidigm). Pre-amplified products (18 cycles)

785 were diluted (1:5) in Universal PCR Master Mix then loaded in a 96.96 Dynamic

786 Array and analyzed on a BioMark Genetic Analysis System (Fluidigm). Data were

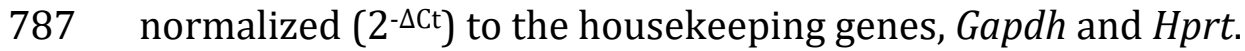

788

789

790

791

792

793

794

Primers used for mRNA detection in total skin samples (TaqMan references)

Acta2 (Mn00808218_g1); Areg (Mm00437583_m1); Ccl1 (Mm00441236_m1); Ccl2 (Mm00441242_m1); Ccl3 (Mm00441259_g1); Clc4(Mm00443111_m1); Clc6 (Mm01302419_m1); Ccl8 (Mm01297183_m1); Cd36 (Mm00432403_m1);

795 (Mm00801666 g1); Col3a1 (Mm00802305 g1); Cxcl2 (Mm00436450 m1); Cxcl9

796 (Mm00434946_m1); Cxcl10 (Mm00445235_m1); Des (Mm00802455_m1); Egr1 
(Mm00656724_m1); Epas (Mm01236112_m1); Fam19a4 (Tafa4,

798 Mm00623620_m1); Flt3 (Mm00439016_m1); Gf1r (Mm00616224_m1); Ifnb1

799 (Mm00439552_s1); Il1b (Mm00434228_m1); Il1r1 (Mm00434237_m1); II4

800 (Mm00445259_m1); Il12a (Mm00434169_m1); Il13 (Mm00434204_m1); Il15 801 (Mm00434210_m1); Il17a (Mm00439618_m1); Il22 (Mm01226722_g1); Il23a 802 (Mm00518984_m1); Irf3 (Mm01203177_m1); Irf7 (Mm00516791_g1); Irf8 803 (Mm00492567_m1); Klf2 (Mm00500486_g1); Klf4 (Mm00516104_m1); Klf5 804 (Mm00456521_m1); Mmp2 (Mm00439498_m1); Mmp9 (Mm00442991_m1); 805 Mmp10 (Mm01168399_m1); Mmp12 (Mm00500554_m1); Mpo 806 (Mm00447885_m1); Mtor (Mm00444968_m); Nos2 (Mm00440502_m1); Nr4a1 807 (Mm01300401_m1); Nr4a3 (Mm00450071_g1); Pdgfra (Mm00440701_m1); 808 Pdgfrb (Mm01262485_m1); Piezo1 (Mm01241544_g1); Piezo2 809 (Mm01262422_g1); Plod2 (Mm00478767_m1); Pparg (Mm00440940_m1); 810 Retlna (Mm00445109_m1); Ripk1 (Mm00436360_m1); Serpinb9 811 (Mm00777163_m1); Tac1 (Mm01166994_g1); Tgfrb2 (Mm03024091_m1); Tlr3 812 (Mm01207402_m1); TIr4 (Mm00445273_m1); Tlr7 (Mm04933178_g1); Tlr9 813 (Mm07299609_m1); Tp53 (Rn00755717_m1); Gapdh (Mm99999915_g1); Hprt 814 (Mm03024075_m1).

815

816 Protein extraction from tissue

817 Mice were euthanized and their ears were snap-frozen in liquid nitrogen and then 818 transferred into mechanical dissociation tubes (Lysis Matrix A, MPbio) containing 819 a commercial lysis buffer (T-Per Tissue Protein Extraction Reagent, 820 Thermofisher) with protease inhibitor cocktail (Halt Protease Inhibitor 100X, 821 Thermofisher). Proteins extraction was performed by incubating each gram of tissue with $20 \mathrm{ml}$ of lysis buffer. Tissues were dissociated using a 3 cycles program 823 of FastPrep-24 5G (MPbio). After two centrifugations at $16000 \mathrm{~g}$ for 30 minutes, 824 the supernatants were filtered (on $70 \mu \mathrm{m}$ cell strainers, Startedt) and stored at $82580^{\circ} \mathrm{C}$ until use.

826

Generation of recombinant mouse TAFA4

828 Mouse TAFA4 recombinant protein was produced in E. coli by Pure Biologics 829 (https://purebiologics.pl). The endotoxin levels of the purified protein, measured 830 by the LAL (Limulus Amoebocyte Lysate) method, was $<0.0005 \mathrm{EU} / \mu \mathrm{g}$. The 831 recombinant protein included a Histidine (His)-Tag in the C-Terminal part of the 832 protein allowing its detection by anti-His antibodies. The sequence of this 833 recombinant mTAFA4 is:

834 MHLIKPGTCEVVAVHRCCNKNRIEERSQTVKCSCFPGQVAGTTRAQPSCVEAAIV 835 IEKWWCHMNPCLEGEDCKVLPDSSGWSCSSGNKVKTTKVTRGGGSGLEHHHHHH 
838 Anti-TAFA4 monoclonal antibodies (mAbs) were generated by MImAbs

839 (https://www.mimabs.org). Briefly, three Wistar rats were immunized with the 840 recombinant mouse TAFA4 protein $(100 \mu \mathrm{g}, 3$ IP injections followed by a final 841 boost). After immunization, spleen cells were harvested and fused to the myeloma 842 cell line X63-Ag8-656. Hybridoma were seeded in methylcellulose semi-solid 843 medium with CloneDetect agent (Molecular Devices \#K8240) and secreting 844 monoclonal IgG hybridomas were picked by ClonePix2 (Molecular Devices) to 845 liquid medium 96 wells plates. Hybridoma clones producing anti-TAFA4 846 antibodies were selected using an ELISA screen. The specificity of the antibodies 847 for the native TAFA4 protein was assessed by immunofluorescent staining on 848 mouse DRG from WT (positive control) or Tafa4-KO (negative control) mice.

\section{Sandwich ELISA for TAFA4 quantification}

851 To quantify TAFA4 protein, sandwich enzyme-linked immunosorbent assay 852 (ELISA) has been developed by MImAbs (https://www.mimabs.org). Briefly, anti853 TAFA4 antibodies (clone 33F8, $50 \mu \mathrm{l}, 2 \mu \mathrm{g} / \mathrm{ml}$ ) diluted in a $\mathrm{Na}_{2} \mathrm{CO}_{3} / \mathrm{NaHCO}_{3}$ buffer 854 (Sigma, \#C3041-100CAP, pH = 9.6) were coated on 96 well MAXISORP plates 855 (Sigma \#M5785-1CS) and incubated overnight at $4^{\circ} \mathrm{C}$. Plates were then washed 3 856 times in a washing buffer (PBS/0.1\% Tween-20) and blocked with in $300 \mu \mathrm{l}$ of 857 StartingBlock blocking buffer (Thermofisher, \#37542). Samples and serial 858 dilutions of mTAFA4 (to establish the standard curve) were incubated $1.30 \mathrm{~h}$ at 859 room temperature (RT). After 3 wash, anti-TAFA4 biotinylated antibodies (clone $8601 \mathrm{D} 8,50 \mu \mathrm{l}, 2 \mu \mathrm{g} / \mathrm{ml}$ ) were incubated for $1.30 \mathrm{~h}$ at RT. After 3 wash, HRP-Conjugated 861 Streptavidin (Thermofisher, \#N100) was added, washed 3 times before adding the 862 substrate solution TMB (Interchim, \#UP664781). After 10 additional minutes, the 863 reaction was stopped with a solution of $\mathrm{HCl}(1 \mathrm{M})$ and the absorbances (at $450 \mathrm{~nm}$ 864 and $620 \mathrm{~nm}$ ) of each well were measured. The specific signal obtained for WT ear 865 skin samples was determined by subtracting the background signal obtained with 866 skin samples from Tafa4-KO mice. 
Ear skin samples were homogenized and proteins extraction was performed as described above. Cytometric Bead Array (CBA) Flex Set kit (BD Bioscience) was

871 used to quantify chemokine ligand 1 (CXCL1), tumor necrosis factor-alpha $872(\mathrm{TNF} \alpha)$, interleukin 6 (IL-6), interleukin 1 beta (IL-1 $\beta$ ), monocyte 873 chemoattractant protein 1 (CCL2/MCP1) and the chemokine (C-C motif) ligands 4 874 (CCL4). For IL-10, an enhanced sensitivity Cytometric Bead Array (CBA) Flex Set 875 kit (BD Bioscience) was used. Samples were analyzed on a Canto II flow cytometer 876 with FCAP Array TM Software (BD Bioscience) following manufacturer's 877 instructions.

\section{$\underline{\text { Statistical analysis }}$}

880 All the results are expressed as mean +/- SEM. Statistical analysis were performed 881 using the GraphPad Prism for Windows software. Statistical analysis was 882 performed using a one-way ANOVA with Tukey's multiple comparisons test 883 throughout the study. A two-way ANOVA test was used to compare two or more 884 than two groups in kinetic experiments after UV exposure, involving repeated 885 measures on the same animals over time. Difference were considered significant 886 as following: ${ }^{*} \mathrm{p}<0.05 ;{ }^{* *} \mathrm{p}<0.01 ;{ }^{* * *} \mathrm{p}<0.001$; and ${ }^{* * * *} \mathrm{p}<0.0001$.

\section{Additional references:}

889

89034 Madan, R. et al. Nonredundant roles for B cell-derived IL-10 in immune counterregulation. J Immunol 183, 2312-2320, doi:10.4049/jimmunol.0900185 (2009).

35 Reber, L. L. et al. Imaging protective mast cells in living mice during severe contact hypersensitivity. JCI Insight 2, doi:10.1172/jci.insight.92900 (2017).

36 Misharin, A. V. et al. Monocyte-derived alveolar macrophages drive lung fibrosis and persist in the lung over the life span. $J$ Exp Med 214, 2387-2404, doi:10.1084/jem.20162152 (2017).

37 Hoeffel, G. \& Ginhoux, F. Ontogeny of Tissue-Resident Macrophages. Front Immunol 6, 486, doi:10.3389/fimmu.2015.00486 (2015).

38 Renthal, W. et al. Transcriptional Reprogramming of Distinct Peripheral Sensory Neuron Subtypes after Axonal Injury. Neuron 108, 128-144 e129, doi:10.1016/j.neuron.2020.07.026 (2020).

39 Tamoutounour, S. et al. Origins and functional specialization of macrophages and of conventional and monocyte-derived dendritic cells in mouse skin. Immunity 39, 925938, doi:10.1016/j.immuni.2013.10.004 (2013).

40 Gentek, R. et al. Hemogenic Endothelial Fate Mapping Reveals Dual Developmental Origin of Mast Cells. Immunity 48, 1160-1171 e1165, doi:10.1016/j.immuni.2018.04.025 (2018). 
931 reviewed and provided input on the manuscript. (2019).

\section{Acknowledgments}

\section{Author contributions}

41 Serhan, N. et al. House dust mites activate nociceptor-mast cell clusters to drive type 2 skin inflammation. Nat Immunol 20, 1435-1443, doi:10.1038/s41590-019-0493-z

We thank Vincent Feuillet for critical reading of the manuscript and for helpful comments and discussions. We thank Justine Galluso for mouse breeding and genotyping. We thank the Centre d'Immunologie de Marseille-Luminy (CIML) mouse house and core cytometry, imaging and histology facilities. The S. U. laboratory received funding from the European Research Council (ERC) under the European Union's Horizon 2020 research and innovation program, under grant agreement No. 648768; and from the Agence Nationnale de la Recherche (ANR) (No. ANR-14-CE140009-01) and from the Fondation pour la Recherche Médicale (FRM, No. ECO201906009090). This work was also supported by institutional grants from INSERM, CNRS, Aix-Marseille University and Marseille-Immunopole to the CIML.

G. H., G. D., A. Ro., R. R., P. V. B., A. B., designed and performed experiments and analyzed data. J.G. did the t-SNE analysis. C. L. and L.C. performed histological analysis. A.M. and A. Re. provided the GINIP-DTR and TAFA4-KO mice, reagents as well as important guidance for the characterization of sensory neurons. S. U. and G. H. designed the study, supervised experiments and co-wrote the manuscript. All authors

\section{Competing interests}


934 S.U., G.H, G.D. and A.M. through Inserm transfert have filed a provisional 935 international patent application WO2020/064907 on the clinical use of TAFA4. All 936 other authors declare no competing interests.

Data availability

939 All data supporting the findings of this study are found within the manuscript and its 940 Supplementary Information. Source data are provided with this paper.

941

942

943

944

945

946

947

948

\section{Extended data legends}

\section{Extended data figure 1: UV overexposure triggers a sequence of skin} inflammation and repair over 35 days and GINIP $^{+}$neurons activation

a, Heatmap representing the expression kinetics of genes involved in skin inflammation and repair over time (days) pi. Ears from WT mice were collected before UV irradiation (D0) or at D3, D7, D14 and D35 pi and total extracted RNA were analyzed by Fluidigm. Genes encoding proinflammatory cytokines and chemokines were expressed at D3 and D7 delineating the inflammatory phase (red cluster). Pro-repair genes were upregulated at D14 and D35 (green cluster) delineating the resolution/remodeling phases. The gene Fam19a4 encoding TAFA4 is highlighted in yellow ( $\mathrm{n}=4-8$ mice per group). b, Sensory neurons from C2/C3 DRGs innervate the ear skin. The fluorescent tracer DiI was injected intradermally in the right ears of WT mice and left ears were injected with PBS ( $\mathrm{n}=3$ independent DRGs per group). c, The trigeminal ganglia (TG) and cervical DRG (C2 to C5) were collected 48h post-injection and analyzed by fluorescent microscopy. d, Quantification of $\mathrm{DiI}^{+}$neurons per DRG fields of view; PBS-injected control side (blue) or DiI-injected side (red). e, Additional representative confocal 
961 images (related to main figure 1c) of C2 DRGs labeled for GINIP (red), CGRP (blue)

962 and ATF3 (green), from unexposed (left) and UV-exposed mice at D3 pi (right). f, 963 Quantification of total $\mathrm{GINIP}^{+}$and $\mathrm{CGRP}^{+}$neurons per DRGs (left) and total $964 \mathrm{CGRP}^{+} \mathrm{ATF}^{+}$and $\mathrm{GINIP}^{+} \mathrm{ATF}^{+}$(right) from unexposed (blue) and UV-exposed mice 965 at D3 pi (red), ( $\mathrm{n}=8$ mice per group). All data are representative for at least two 966 independent experiments and presented as means \pm SEM. One-way ANOVA with 967 Tukey's multiple comparisons test (ns: non-significant; ${ }^{*} p<0.05 ;{ }^{* *} p<0.01 ;{ }^{* * *} p<$ $9680.001)$.

969

970

971 
Extended data figure 2: Conditional ablation of GINIP ${ }^{+}$neurons in GINIP-DTR mice and skin histopathological analysis after UV exposure.

a, Representative confocal images for DAPI (blue) and GINIP (green) staining of C3 DRGs from control DTR-control (upper panels) and GINIP-DTR (lower panels) mice, 10 weeks post-DT treatment. b, Absolute number of GINIP ${ }^{+}, \mathrm{CGRP}^{+}, \mathrm{TAFA}^{+}, \mathrm{TH}^{+}$ and IB4 $4^{+}$DRG neurons were quantified in DTR-control (blue) and GINIP-DTR mice (red), ( $n=4-6$ independent DRG per group). c, Representative confocal images for Beta3-tubulin (blue) and GINIP (green) staining, of mouse ear skin sections as in a. d, 980 scratching episodes were monitored during 30 min at each time point pi indicated $(n=6-$ 98110 mice per group). e, Representative H\&E staining of ears from DTR (left) and GINIP982 DTR (right) mice at D35 pi. Histopathological analysis for leukocyte infiltration (f), 983 epidermal thickness (g), fibrosis (h) and fibrosis extension (i) ( $\mathrm{n}=5$ mice per group) 984 related to the main Fig. 1g-j. Criteria used for the histopathological scoring are 985 described in the Article Methods. j, Representative Masson trichrome (upper panel) 986 and H\&E staining (lower panel) of back skin from DTR-control (left) and GINIP-DTR 987 (right) mice at D35 pi. All data are representative for at least two independent 988 experiments and presented as mean \pm SEM. One-way ANOVA with Tukey's multiple 989 comparisons test (ns: non-significant; ${ }^{*} p<0.05 ;{ }^{* *} p<0.01 ; * * * p<0.001$ ). 

exposure.

993 a, Flow cytometry gating strategy using CD11b, CD11c, CD64, MHC-II, CD103, c994 kit, Ly6G and CD24 marker expression. Dendritic cells (DC; CD11 $\mathrm{c}^{+} \mathrm{MHCII}^{+}$), mast

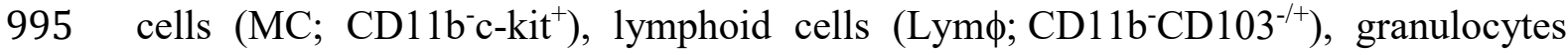
$\left(\mathrm{CD} 11 \mathrm{~b}^{+} \mathrm{CD} 24^{+}, \mathrm{Ly}_{6 \mathrm{G}}^{+}\right)$and the monocyte/macrophage compartment $(\mathrm{Mo} / \mathrm{M} \phi$; CD11 $\left.\mathrm{c}^{-} \mathrm{MHCII}{ }^{-\mathrm{CD}} 11 \mathrm{~b}^{+} \mathrm{CD} 24^{-} \mathrm{Ly}_{6 \mathrm{G}}{ }^{-\mathrm{CD}} 64^{\mathrm{int} /+}\right)$ are shown. b, Absolute numbers per mg 998 of skin for DC, MC, Lym $\phi$, Gr and Mo/M $\phi$ populations at D14 pi in DTR-control (blue) or GINIP-DTR (red) mice, (n=8 mice per group). c, Gating strategy for skin dendritic cell subsets $\left(\mathrm{CD}_{11 \mathrm{c}^{+} \mathrm{MHCII}}\right)^{+}$and monitoring of Langerhans cells (LC; 1001 EpCAM $^{+}$Langerin $^{+}$), (n=5-8 mice per group). d, FACS plot for LC (left) and 1002 quantification (right) in DTR-control and GINIP-DTR mice, unexposed (No UV) or at 1003 D14 pi (UV), (n=5-10 mice per group). e, Percentage of LC among DC over time postUV exposure in WT and Tafa4 KO mice. f, Representative flow cytometry analysis of skin immune cells and DRG neurons for the expression of DTR in GINIP-DTR mice. g, Confocal analysis of whole mount ear skin from Nav1.8-RFP mice stained for $1007 \mathrm{GINIP}^{+}$neurons and CD206 ${ }^{+}$dermal resident M $\phi$. Hair follicles (HF) are highlighted 1008 within white dashed squares. In the skin, Nav1.8 expression (RFP) is restricted to sensory neuron axonal extensions. In DRG (f), DTR expression is restricted to neuronal 1010 cellular bodies in DRGs. These markers were not detected in immune cells. All data 1011 are representative for at least two independent experiments and presented as mean \pm 1012 SEM. One-way ANOVA with Tukey's multiple comparisons test (ns: non-significant; 1013 $\left.{ }^{*} p<0.05 ; * * p<0.01 ; * * p<0.001\right)$. 
1017 Extended data figure 4: Flow cytometry and t-SNE analysis of skin 1018 Monocyte/Macrophage subsets in GINIP-DTR mice.

1019 a, Classical flow cytometry gating strategy for Monocyte and Macrophage (Mo/M $\phi)$ 1020 subsets in the skin. b, t-SNE analysis including CCR2, Ly6C, MHC-II, CD64, CD206 1021 and Tim4 markers was performed to cluster Mo/M $\phi$ populations at day 14 pi in the skin 1022 of GINIP-DTR and DTR-control mice (See also Article methods for t-SNE analysis). 1023 c, Representative plots for CCR2 ${ }^{-/+} \mathrm{CD} 206^{-}$monocyte subsets on D3, D7 and D14 pi in 1024 the skin of control (DTR) or GINIP-DTR mice. d, Absolute numbers of Ly6C ${ }^{+}$Mo, Int. 1025 Mo, PMo and MoDC subsets, per mg of ear skin ( $\mathrm{n}=9-13$ mice per group) as in c. e, 1026 Representative FACS plots for CD206 ${ }^{+}$dermal resident $\mathrm{M} \phi$ as in c. f, Absolute numbers 1027 of DN and MHC $\mathrm{II}^{+} \mathrm{M} \phi$ subsets, per mg of ear skin (n=9-13 mice per group) as in c. 1028 g, Representative FACS plots for CD206 ${ }^{+}$dermal resident M $\phi$ subsets at D14 pi in back 1029 skin from control (DTR) or GINIP-DTR mice. $\mathbf{h}$, Absolute numbers of M $\phi$ subsets, 1030 per mg of back skin ( $\mathrm{n}=12$ mice per group) from DTR-control (gray) or GINIP-DTR 1031 (colored) mice, at D14 pi. All data are representative for at least two independent 1032 experiments and presented as mean \pm SEM. One-way ANOVA with Tukey's multiple 1033 comparisons test (ns: non-significant; $* p<0.05 ; * * p<0.01 ; * * * p<0.001$ ). 

release TAFA4 in the skin.

1037 a, Representative confocal images of whole mount ear skin from WT mice stained with 1038 an anti-Beta3-tubulin (blue) and anti-GINIP (green) antibodies. GINIP ${ }^{+}$lanceolate 1039 barrel structures innervating hair follicles (HF) are highlighted within dashed squares.

1040 b, Confocal analysis as in a with an additional anti-TH antibody staining (red) shows $1041 \mathrm{GINIP}^{+} \mathrm{TH}^{+}$C-LTMR axon terminals reaching hair follicle. c, Schematically, GINIP ${ }^{+}$ 1042 neurons consist of two subsets of sensory neurons projecting in the inter-follicular 1043 regions of the epidermis as free nerve endings $\left(\mathrm{IB}^{+}{ }^{+}\right)$and hair follicles as C-LTMRs 1044 (TAFA4 $\left.^{+}\right)$. d, Confocal images of DRG sections from control WT (left) or Tafa4-KO 1045 (right) mice after immunofluorescent (IF) staining with an anti-GINIP (green) an anti1046 TAFA4 antibody (1D8; red) and DAPI (blue). e, Additional confocal images of C3 1047 DRGs (IF) stained with the isolectin B4 (blue), anti-TAFA4 (1D8, red), and ATF3 1048 (green) antibodies, in WT mice (related to main Figure 3), unexposed (left) or at D3 1049 pi (right). f, Absolute number of TAFA4 ${ }^{+}$and $\mathrm{IB}^{+}{ }^{+}$neurons (left; $\mathrm{n}=6-12$ mice per 1050 group) and absolute number of $\mathrm{TAFA}^{+} \mathrm{ATF}^{+}$and $\mathrm{IB}^{+} \mathrm{ATF}^{+}$neurons per $\mathrm{DRG}$ 1051 (right) from unexposed (blue) and exposed D3 pi (red) WT mice, (n=8 mice per group). g, DAPI staining (blue) in situ hybridization (ISH) for Tafa4 mRNA (red) in DRG as 1053 in d. h, Quantification of TAFA4 levels (ELISA) in DRGs from WT mice unexposed 1054 (D0) to D14 pi. i, Tafa4 mRNA expression (RT-qPCR) in peripheral tissues and DRGs 1055 ( $\mathrm{n}=3$ mice per time point; n.d: not determined). j, Quantification of Tafa4 mRNA 1056 expression in PBMC, BM, BMDM and sorted CD206 ${ }^{+}$dermal M $\phi$ compared to DRGs 1057 ( $\mathrm{n}=3$ independent samples). $\mathbf{k}$, Tafa4 mRNA expression in C3 DRGs from D0 to D35 1058 pi ( $\mathrm{n}=3$ mice per time point). All data are representative for at least two independent 1059 experiments and presented as mean \pm SEM. One-way ANOVA with Tukey's multiple 
1060

comparisons test except $\mathbf{h}$, Kruskal-Wallis (ns: non-significant; ${ }^{*} p<0.05 ;{ }^{* *} p<0.01$;

$1061 * * * p<0.001)$. 
Extended data figure 6: Tafa4 regulates skin inflammation after UV exposure but does not affect Treg and Mast cell IL-10 production.

a, Changes in ear skin thickness over time pi in WT control (blue) and Tafa4-KO (red) mice ( $n=9$ mice per group). $\mathbf{b}$, Representative H\&E images of ears from WT (left) or Tafa4-KO (right) mice at D35 pi and histopathological scoring for leukocyte 1067 infiltration, epidermal thickness, fibrosis, fibrosis extension and then c, cumulative 1068 fibrosis score and total pathological scores ( $\mathrm{n}=12$ mice per group). See detailed scoring in Article methods. d, IL-6, IL-1 $\beta$, and chemokines CCL2, CXCL1 and CCL4 protein 1070 levels (measured by CBA) and Il10 mRNA level, in ear skin from WT (blue) or Tafa41071 KO (red) mice from D0 to D35 pi (n=3-11 mice per group and time point). e, FACS 1072 analysis and detection of IL-10 $0^{+}$immune cells from control (WT; gray) and IL-10-GFP (red) mice using an anti-GFP antibody and f, using an anti-IL-10 antibody, in skin DC 1074 subsets, Lym $\phi$ cells, monocytes, mast cells (MC) and M $\phi$ subsets in control (IL-10 ${ }^{\text {gfp/gfp }}$ mice, gray) and WT (blue) mice. g, Gating strategy for Tregs $\left(\mathrm{CD} 4^{+} \mathrm{TCR} \beta^{+} \mathrm{FoxP} 3^{+}\right)$ and $\mathbf{h}$, absolute number per mg of ear skin before (D0) or pi, in DTR-control (blue) or 1077 GINIP-DTR (red) mice ( $\mathrm{n}=9-18$ mice per group and per time point). i, Representative 1078 FACS plots for IL-10 in Tregs from WT (left) or Tafa4-KO (right) mice before (blue) 1079 or pi (red). j, Gating strategy (left) and absolute number (right) of mast cells (MC) per mg of skin from DTR-control (blue) or GINIP-DTR (red) mice, showing MC depletion 1081 (D3) and repopulation (D7) after UV exposure; ( $\mathrm{n}=8-9$ mice per group and time point). $1082 \mathbf{k}$, Representative FACS plots for IL-10 in $\mathrm{Tim}^{+} \mathrm{M} \phi$ (blue) and MC (yellow) before 1083 (No UV, top) and at D10 pi (bottom) in WT mice. 1, Representative FACS plot for IL108410 in MC in WT (blue) or Tafa4-KO (red) mice at D10 pi and $\mathbf{m}$, median IL-10 MFI 1085 analysis ( $\mathrm{n}=6-10$ mice per group). All data are representative for at least two 1086 independent experiments and presented as mean \pm SEM. One-way ANOVA with 
Tukey’s multiple comparisons test (ns: non-significant; ${ }^{*} p<0.05 ;{ }^{* *} p<0.01 ;{ }^{* *} p<$ $0.001)$.

Extended data figure 7: TAFA4 promotes IL-10 production by dermal resident M $\phi$ subsets in vivo and directly up-regulates $I 110$ in $\mathrm{M} \phi$ in vitro.

(a), MHC-II ${ }^{+} \mathrm{M} \phi(\mathbf{b})$ and $\mathrm{Tim} 4^{+} \mathrm{M} \phi$ (c) subsets from WT (blue) and Tafa4-KO (red) mice at D10 pi, and (lower panels) median IL-10 MFI quantifications in the respective $\mathrm{M} \phi$ subsets at D3, 7 and 10 pi ( $\mathrm{n}=6-9$ mice per group and time point). d, BMDM, 1096 derived from WT or FPR1-KO bone marrow, were challenged in a migration assay 1097 (transwell) using medium alone $\left(\mathrm{CTL}^{-}\right), \mathrm{MCP}-1\left(\mathrm{CTL}^{+}\right)$or increasing concentrations of 1098 TAFA4. Absolute number of $\mathrm{M} \phi$ in the bottom wells were analyzed by FACS $(\mathrm{n}=3$ 1099 independent samples per group). e, IL6 mRNA level in ThioM $\phi$ in the presence (green) 1100 or absence (blue) of TAFA4 (See main Fig.4i), (n=6-8 independent sample per group). 1101 f, BMDM as in (d), were activated in vitro by LPS alone (blue) or in the presence of 1102 TAFA4 (green). Gene expression was analyzed by RT-qPCR for Tnfa, Il6 and Ill0 and 1103 compared to non-activated BMDM (CTL), $(n=4-5$ independent sample). All data are 1104 representative for at least two independent experiments and presented as mean $\pm \mathrm{SEM}$. 1105 One-way ANOVA with Tukey's multiple comparisons test $\left({ }^{*} p<0.05 ;{ }^{* *} p<0.01 ;{ }^{* * *} p\right.$ $1106<0.001)$ 
1112 a, Strategy for embryonic progenitor fate mapping using the CX3CR1 ${ }^{\text {CreERT2:R26-YFP }}$

1113 mice. Tamoxifen (Tam) was injected in pregnant females at E16.5 days of pregnancy

1114 and offsprings were analyzed at 6 weeks of age. b, Percentages of $\mathrm{YFP}^{+}$cells in the

1115 indicated cell types ( $\mathrm{n}=5$ mice). LCs: Langerhans cells. c, Experimental scheme for 1116 monocyte tracing using CD45.1 $1^{+} \mathrm{BM}$ shield-irradiated chimeras. d, Percentage of $1117 \mathrm{CD} 45.1$ chimerism within the indicated Mo/M $\phi$ subsets in 2 months old chimeric mice 1118 in the steady state ( $\mathrm{n}=7$ mice per group), or $\mathbf{e}$, after additional 4 months following UV 1119 irradiation (purple) or no UV (gray), ( $\mathrm{n}=5$ mice per group). f, Gating strategy for $1120 \mathrm{Mo} / \mathrm{M} \phi$ analysis in WT CD45.1 BM Chimera before UV exposure (top panels) and 1121 then at D7 pi in WT (middle panels) and in Tafa4-KO (bottom panels) recipient 1122 chimeras. g, Relative expression of the markers CD45.1, Ly6C, CD64, CD206, MHCII 1123 and Tim4 used for t-SNE analysis of skin Mo/M $\phi$ subsets from WT or Tafa4-KO 1124 recipient chimeras (see also Fig. 4a). h, Absolute numbers ( $\mathrm{n}=9$ mice per group) per 1125 mg of ear skin (left) and CD45.1 chimerism levels (right) ( $\mathrm{n}=7$ mice per group) for DN $1126 \mathrm{M} \phi$ and MHC-II ${ }^{+} \mathrm{M} \phi$ in WT (blue) and Tafa4-KO (red) BM-chimeric mice on D7 pi. 1127 i, Representative Annexin-V staining of $\mathrm{DN} \mathrm{M} \phi$ (left), $\mathrm{MHC}-\mathrm{II}^{+} \mathrm{M} \phi$ (middle) and $1128 \mathrm{Tim}^{+} \mathrm{M} \phi$ (right) at D3 pi from DTR (blue) or GINIP-DTR (red) mice. $\mathbf{j}$, median MFI 1129 for annexin- $\mathrm{V}$ in $\mathrm{M} \phi$ subsets as in (i), (n=4 mice per group). $\mathbf{k}$, Representative Annexin$1130 \mathrm{~V}$ as in (i) at D2 pi for WT (blue) or Tafa4-KO (red) mice. 1, Median fluorescence 1131 intensity (MFI) for Annexin-V in M $\phi$ subsets as in (j), $(n=5-6$ mice per group). All data 1132 are representative for at least two independent experiments and presented as mean \pm 
1133 SEM. One-way ANOVA with Tukey's multiple comparisons test (ns: non-significant;

$1134 * p<0.05 ; * * p<0.01 ; * * * p<0.001)$

1135 


\section{KO mice phenotype after UV exposure.}

1138 a, Experimental scheme for in vivo neutralization of IL-10 in WT mice. b, Ear thickness 1139 at D14 pi, of WT (blue) or Tafa4-KO (red) mice treated with an IgG2a isotype control 1140 (iso) and of WT mice treated with anti-IL-10 blocking antibodies (purple), $(\mathrm{n}=10$ mice 1141 per group). c, Absolute numbers of Inflam $\mathrm{M} \phi$ (left) and $\mathrm{Tim}^{+} \mathrm{M} \phi$ (right) in ear skin 1142 of the indicated 3 groups of mice (as in $\mathbf{b}),(n=6-9$ mice per group). $\mathbf{d}$, Representative 1143 FACS plots of Mo/M $\phi$ subsets at D0 and D3 pi in IL-10 ${ }^{\mathrm{wt} / \mathrm{gfp}}$ and IL-10 $\mathrm{gfp} / \mathrm{gfp}$ (IL-10 KO) 1144 mice. e, Absolute number of $\operatorname{Tim} 4^{+} \mathrm{M} \phi$ (left) and Inflam $\mathrm{M} \phi$ (right) per mg of skin at 1145 D7 pi in IL-10 ${ }^{\mathrm{wt} / \mathrm{gfp}}$ (gray) and IL-10 $0^{\mathrm{gfp} / \mathrm{gfp}}$ (colored), (n=8-10 mice per group). f, ear 1146 thickness before (No UV) and at D14 pi (UV) in IL-10 ${ }^{\text {wt/gfp }}$ (blue) and IL-10 gfp/gfp 1147 (purple) mice, ( $\mathrm{n}=9-12$ mice per group). $\mathbf{g}$, Experimental scheme for dermal resident 1148 M $\phi$ depletion: two i.p. injections, at D-3 and D-2, of the CSF-1R blocking antibody 1149 AFS98, are effective to deplete DN, Tim4 ${ }^{+}, \mathrm{MHC} \mathrm{II}^{+}$and Langerhans cells in WT mice. $1150 \mathbf{h}$, Absolute number of $\mathrm{M} \phi$ per mg of ear skin before (CTL) and after AFS98 injection 1151 (colored), ( $\mathrm{n}=13$ mice per group). i, IL-10 protein level detected by CBA in WT (blue) 1152 and Tafa4-KO (red) after isotype control or AFS98 antibody injection ( $\mathrm{n}=4-5$ mice per 1153 group). $\mathbf{j}$, Absolute number of myeloid cell subsets per mg of ear skin after isotype 1154 control or AFS98 antibody injection (colored), $(n=11$ mice per group). $\mathbf{k}$, (left) 1155 Representative FACS plots of $\mathrm{Mo} / \mathrm{M} \phi$ subsets over the time course of $\mathrm{M} \phi$ repopulation 1156 (D0, D2, and D4) after AFS98 injection and complete M $\phi$ depletion (D0) in WT mice, 1157 and, (right) Representative FACS plots at D6 after AFS98 injection in WT (blue) and 1158 Tafa4-KO (red) mice showing the acquisition of Tim4 by monocytes-derived M $\phi$, (see 1159 bottom panels). I, Representative FACS plots of Mo/M $\phi$ subsets at D0 and D3 post-UV 1160 exposure in WT and Tafa4-KO mice after M $\phi$ depletion with the blocking anti-CSF- 
1161 1R Ab (AFS98). Absolute number of Tim4 $4^{+} \mathrm{M} \phi \mathbf{m}$ (left), and Inflam $\mathrm{M} \phi$ (right), per 1162 mg of skin at D7 pi in WT (gray) and Tafa4-KO (colored) treated with an IgG2a isotype 1163 control (Iso+UV), or treated with anti-CSF-1R Ab (AFS+UV), ( $\mathrm{n}=8-9$ mice per group). 1164 n, ear thickness in unexposed mice (No UV), and at D14 pi in WT (blue) and Tafa4$1165 \mathrm{KO}$ (red) mice treated as in $\mathbf{m}$ ( $\mathrm{n}=10$ mice per group). All data are representative for at 1166 least two independent experiments and presented as mean \pm SEM. One-way ANOVA 1167 with Tukey's multiple comparisons test (ns: non-significant; ${ }^{*} p<0.05 ; * * p<0.01$; $1168 * * * p<0.001)$ 

reduce tissue damage in GINIP-DTR mice.

1172 a, Experimental scheme for intradermal adoptive transfer of CD45. $1^{+} \operatorname{Tim}^{+} \mathrm{M} \phi . \mathbf{b}$,

1173 Gating strategy for sorting CD45.1 ${ }^{+}$Inflam $\mathrm{M} \phi$ (pink) and $\mathrm{Tim}^{+} \mathrm{M} \phi$ (cyan). c, ear 1174 thickness for unexposed (No UV) or UV-exposed mice at D7 pi, in DTR (blue), GINIP1175 DTR (red) mice and GINIP-DTR mice injected with Inflam M $\phi$ (pink) or with Tim4 ${ }^{+}$ $1176 \mathrm{M} \phi$ (cyan) mice, ( $\mathrm{n}=7-10$ mice per group). d, Representative images of ears at D7 pi 1177 from each group as in (c). e, Experimental scheme for TAFA4 rescue in Tafa4-KO 1178 mice. f, Representative FACS plot for intracellular staining of IL-10 in $\operatorname{Tim}^{+} \mathrm{M} \phi$ at 1179 D14 pi, in WT mice treated with saline (blue) or Tafa4-KO mice treated with saline 1180 (red) or Tafa4-KO mice treated with TAFA4 (green). g, Model for TAFA4 functions 1181 in vivo after UV exposure: 1) Role of TAFA4 during the inflammatory phase. Skin 1182 overexposure to UV induces the release of the neuropeptide TAFA4 by C-LTMRs. 1183 TAFA4 promotes the production of IL-10 by embryonic-derived dermal resident Tim4 ${ }^{+}$ $1184 \mathrm{M} \phi$. The production of IL-10 is essential for their survival and protect the skin from 1185 over-inflammation. Tissue lesions also induce the recruitment of monocytes in the skin, 1186 where they differentiate into $\mathrm{TNF}^{+}$Inflam $\mathrm{M} \phi$. In the absence of TAFA4 production, 1187 the number of $\mathrm{Tim} 4^{+} \mathrm{M} \phi$ and IL-10 levels are reduced, promoting the expansion of 1188 Inflam Mф. 2) Role of TAFA4 during the resolution phase. The TAFA4/IL-10 axis is 1189 still active, promoting the maintenance of both embryonic- and monocyte-derived IL$119010^{+} \mathrm{Tim}^{+} \mathrm{M} \phi$, which are required for tissue repair. In the absence of TAFA4 1191 production, IL-10 production by the three subsets of $\mathrm{CD}_{206^{+}}$dermal $\mathrm{M} \phi$ is 1192 compromised, leading to persistent inflammation and fibrotic scars. h, Repeated 1193 measure over time of ear thickness changes $(\mu \mathrm{m})$ of WT (blue) and Tafa4-KO (red) 1194 mice treated daily with Imiquimod (IMQ) during six consecutive days and followed up 
1195 to 15 days, ( $\mathrm{n}=5$ mice per group). All data are representative for at least two 1196 independent experiments and presented as mean \pm SEM. One-way ANOVA with 1197 Tukey's multiple comparisons test (ns: non-significant; ${ }^{*} p<0.05 ;{ }^{* *} p<0.01 ;{ }^{* * *} p<$ $11980.001)$.

1199

1200 


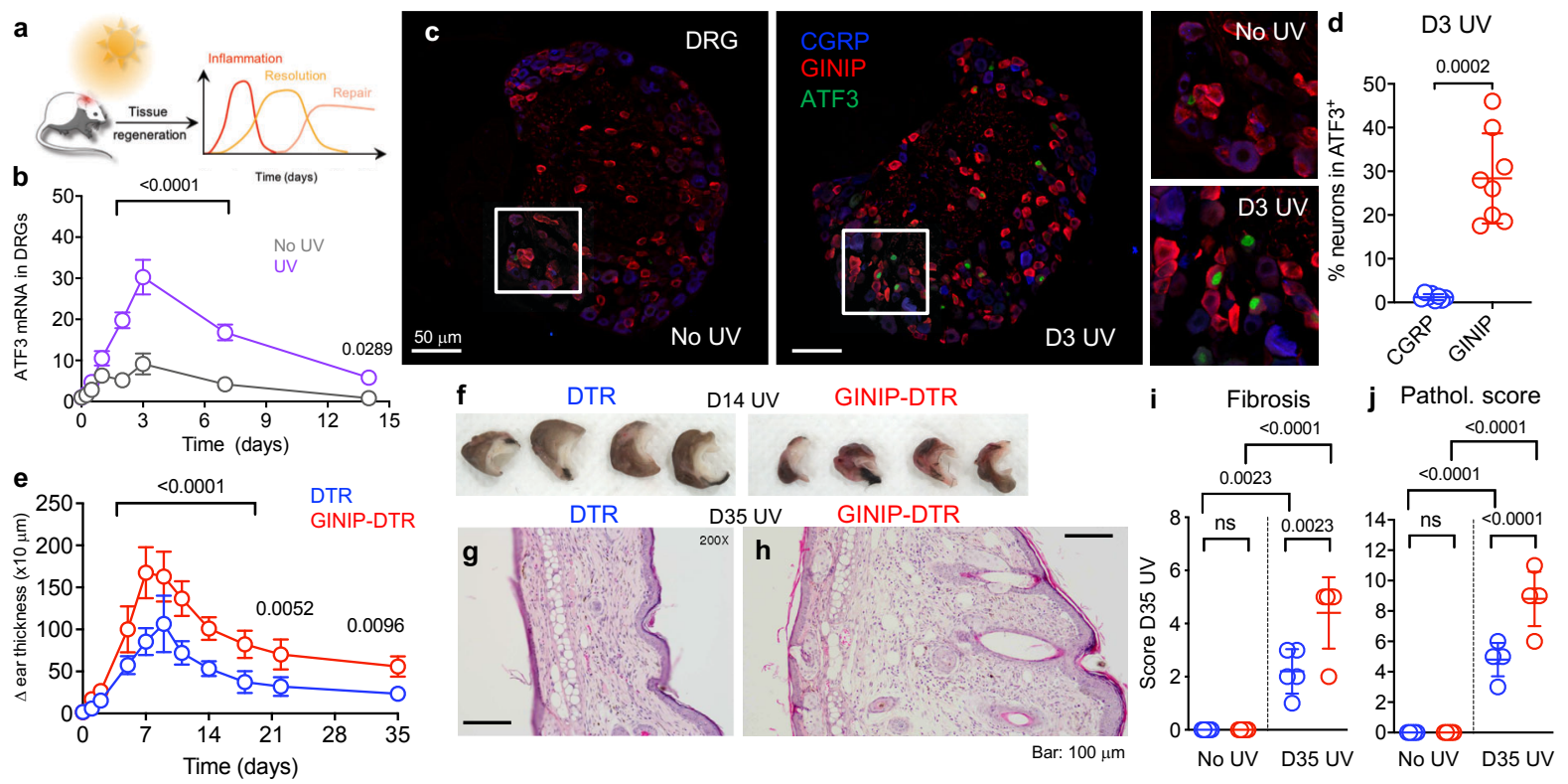



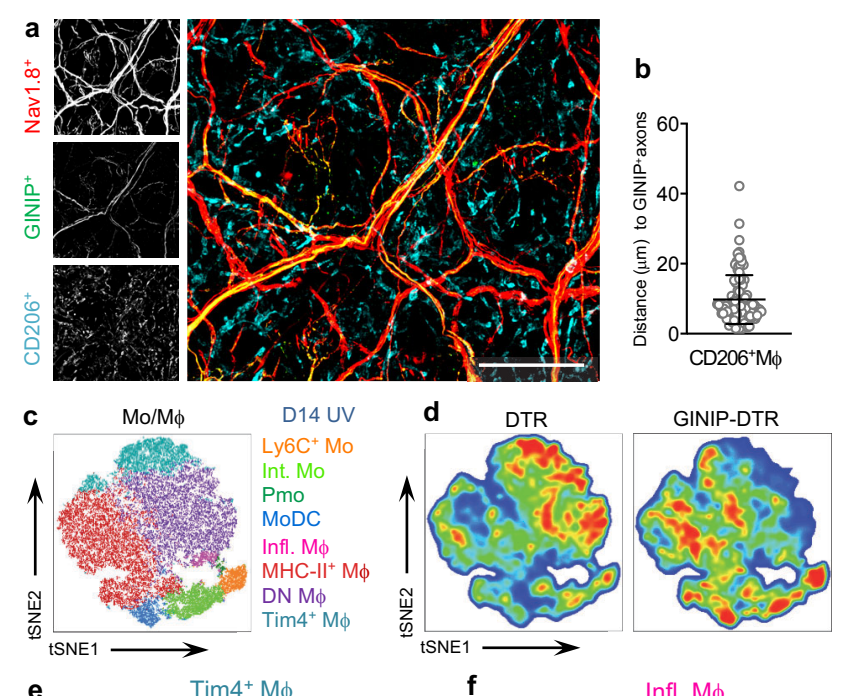

$$
\text { e }
$$
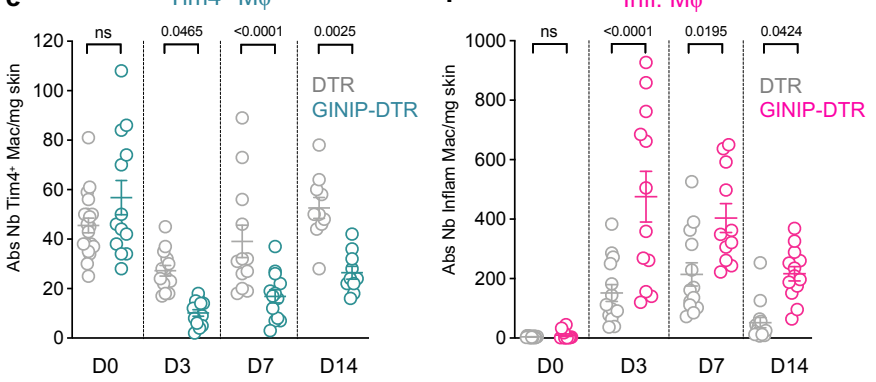


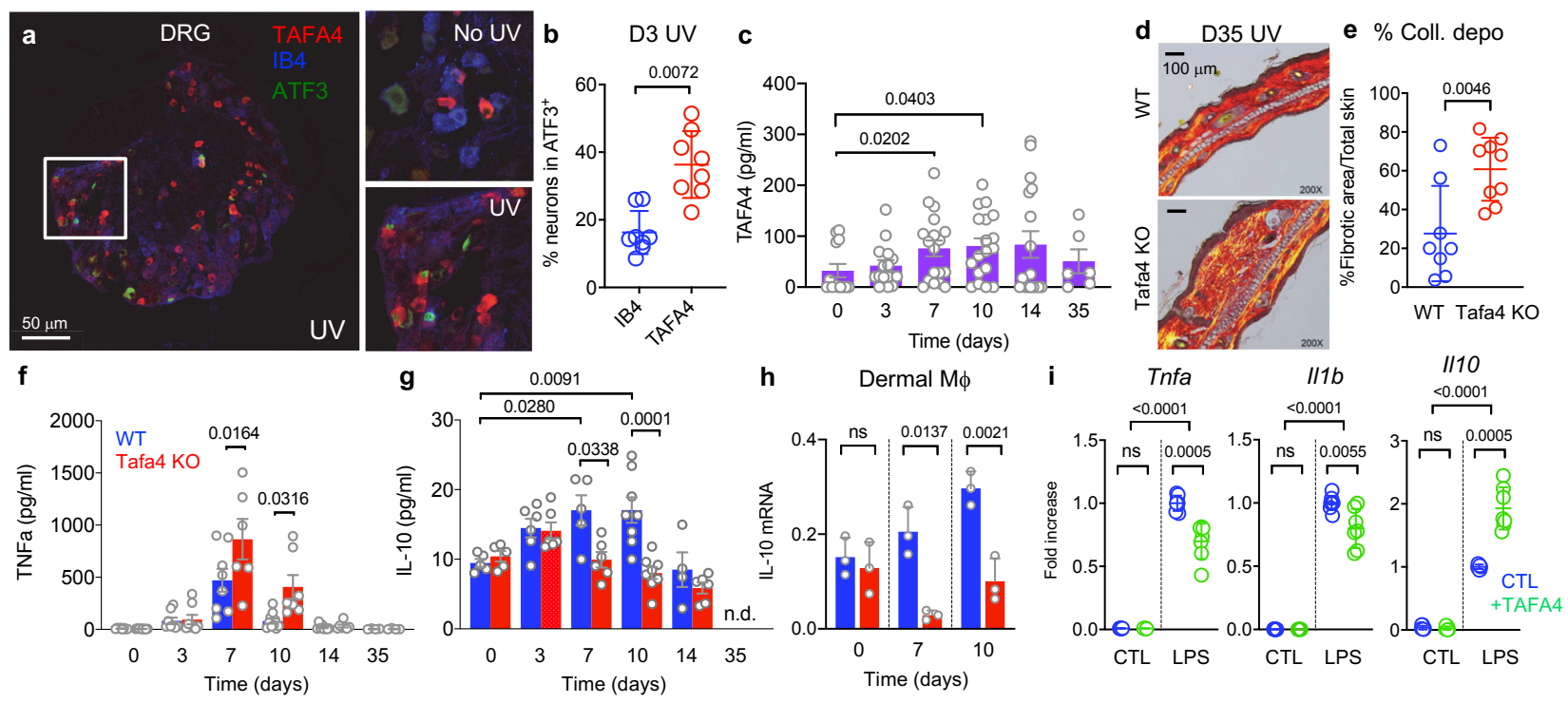



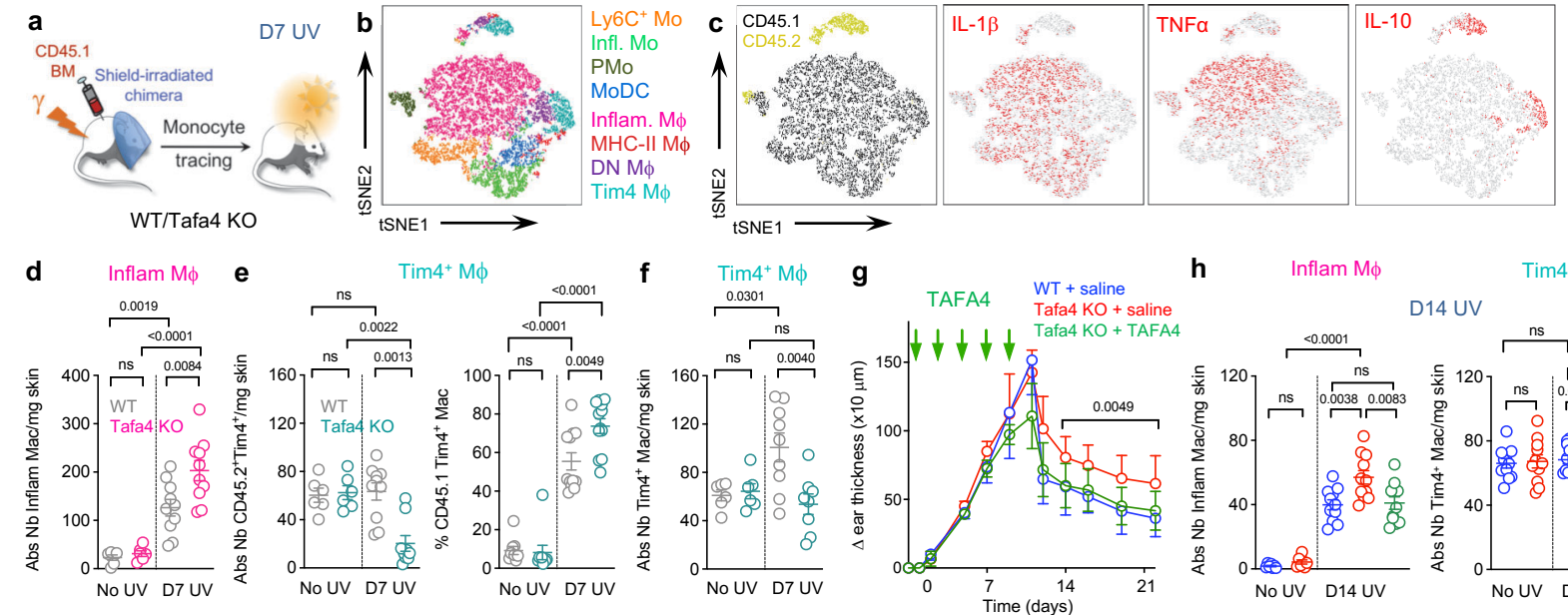

h Inflam $\mathrm{M} \phi \quad \mathrm{Tim}^{+} \mathrm{M} \phi$
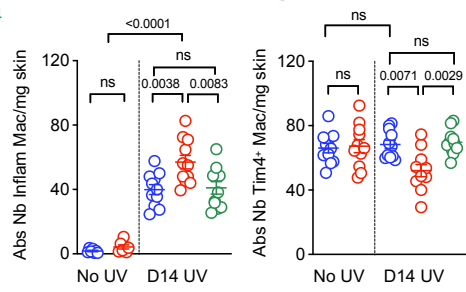


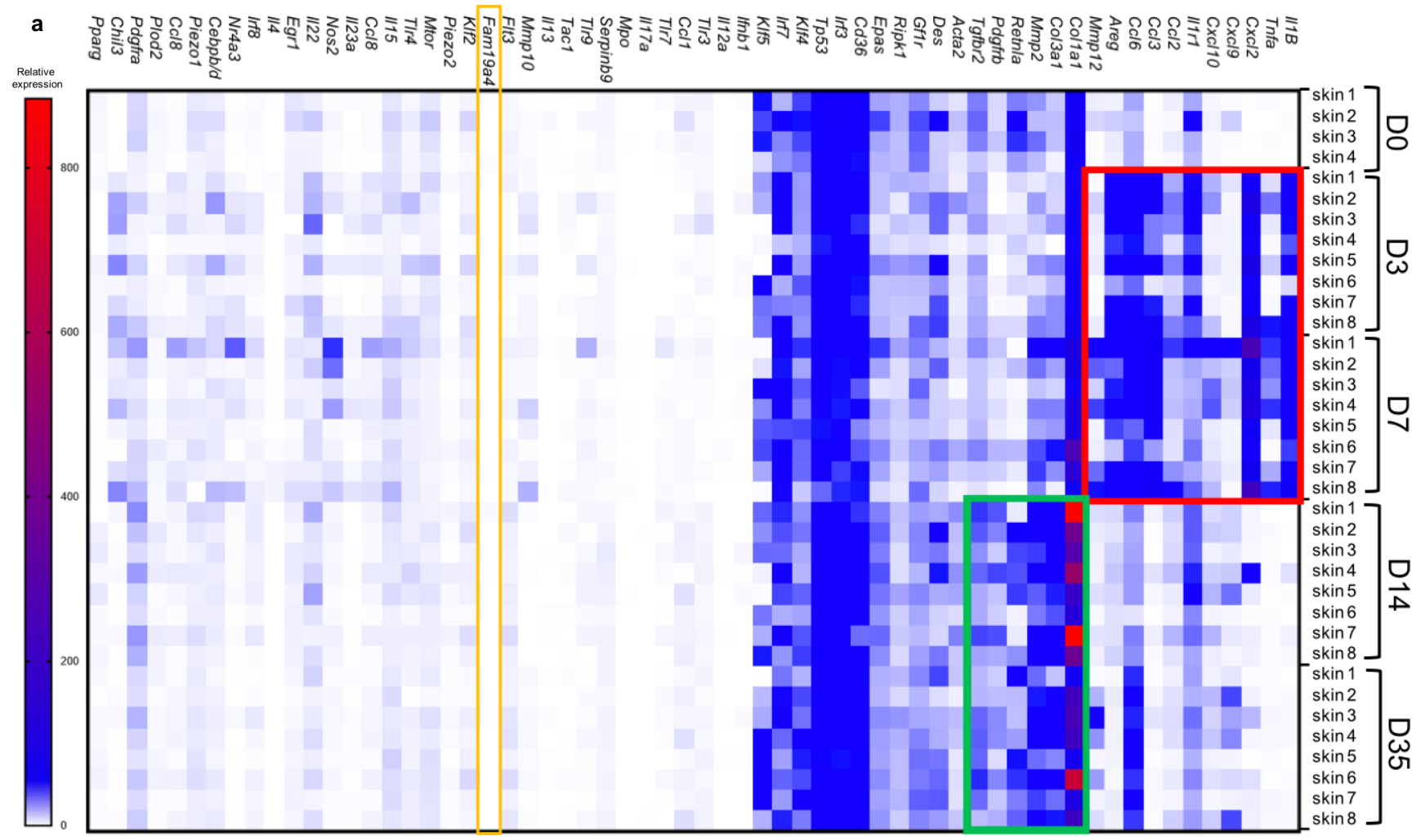

Identification of DRGs innervating the ear skin by Dil retrolabeling

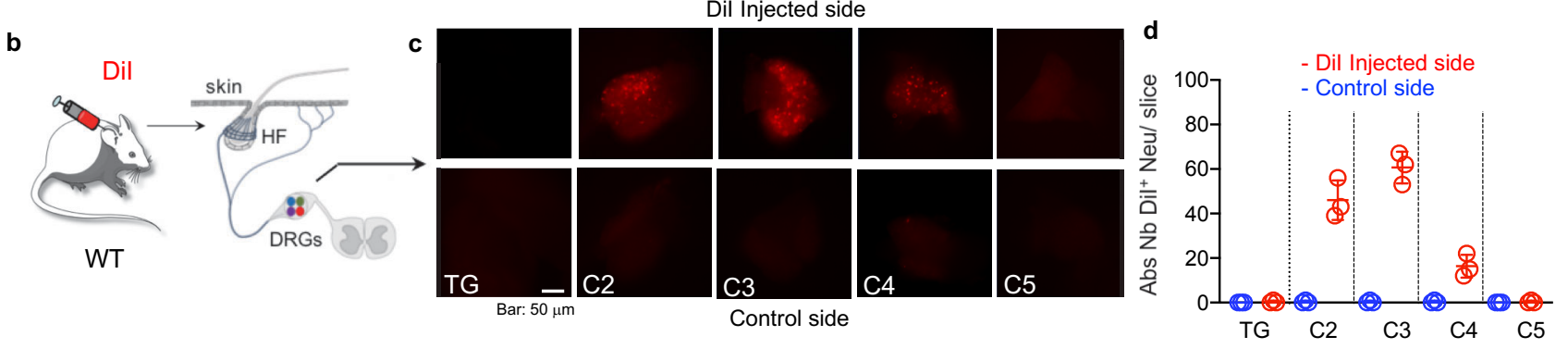

e

UV exposure selectively activate GINIP $^{+}$neurons in C2/C3 DRGs
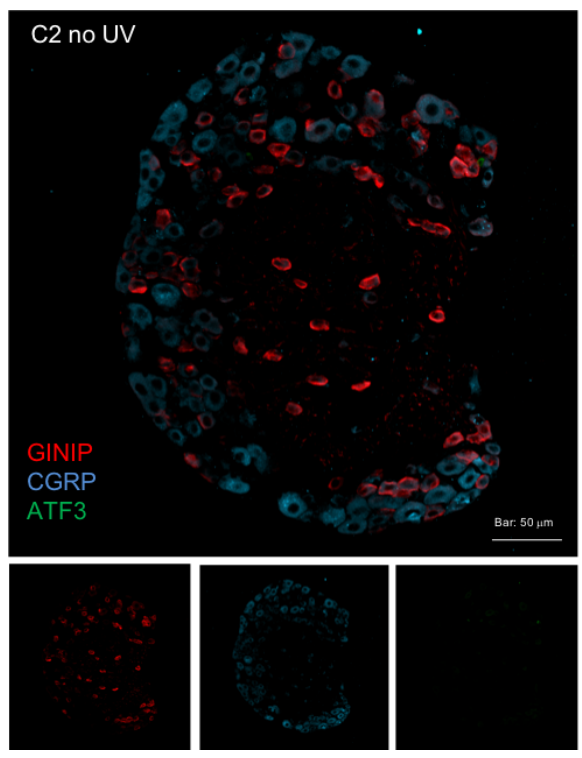
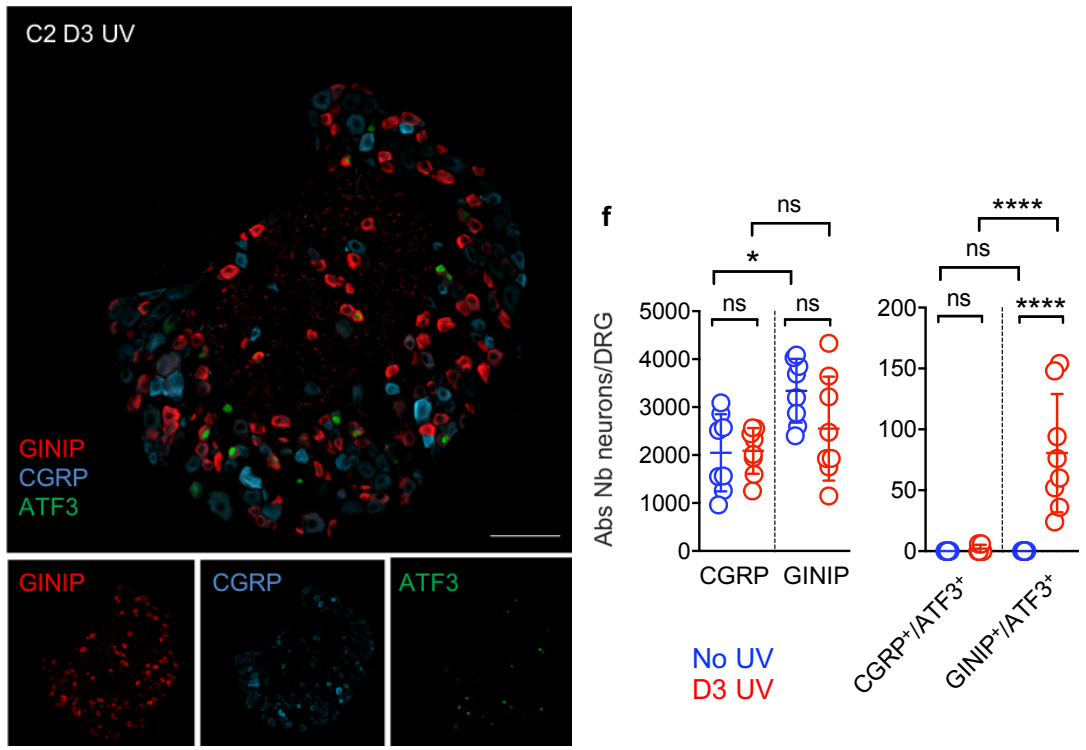

No UV

D3 UV

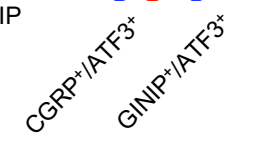



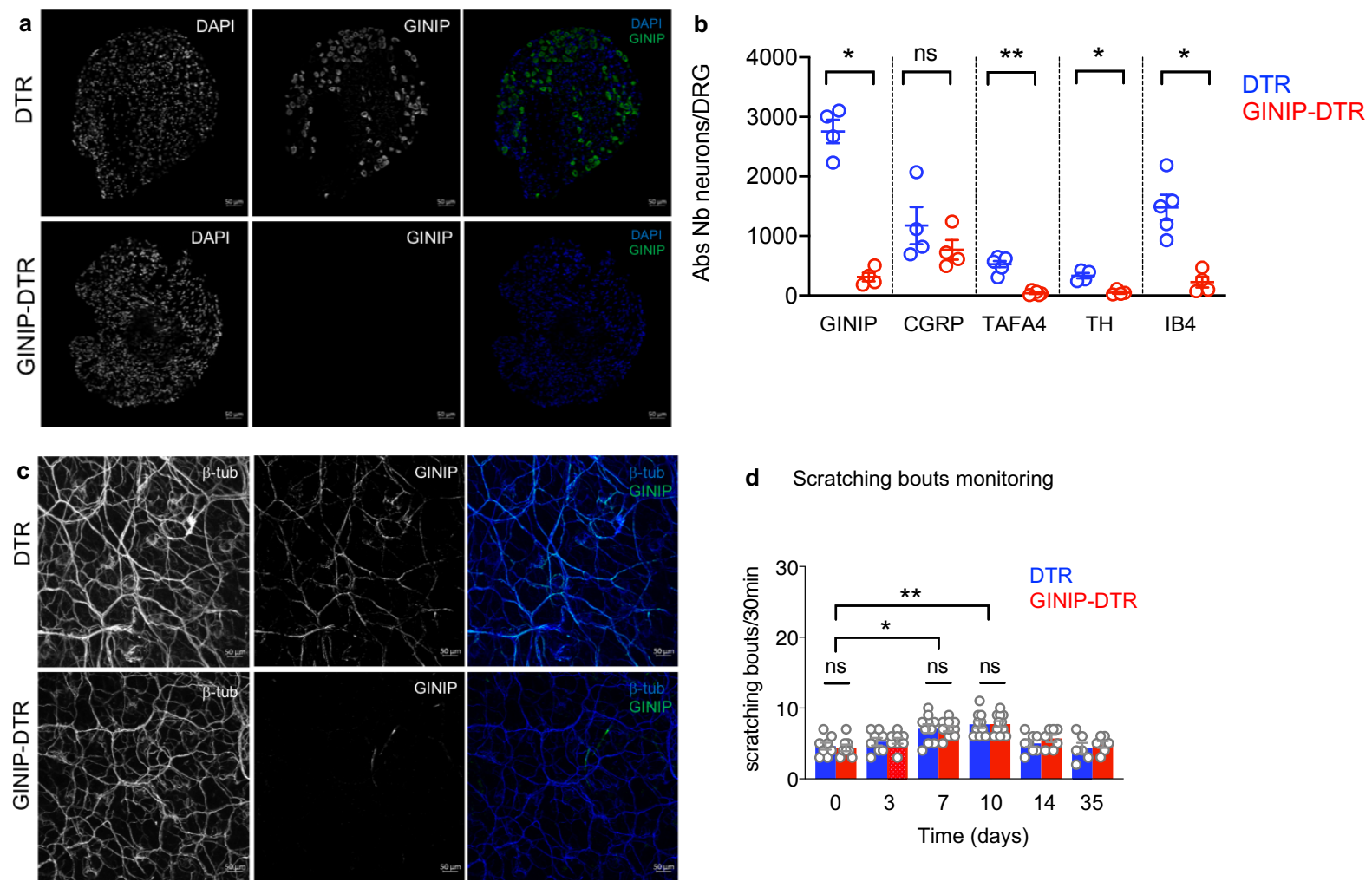

d Scratching bouts monitoring
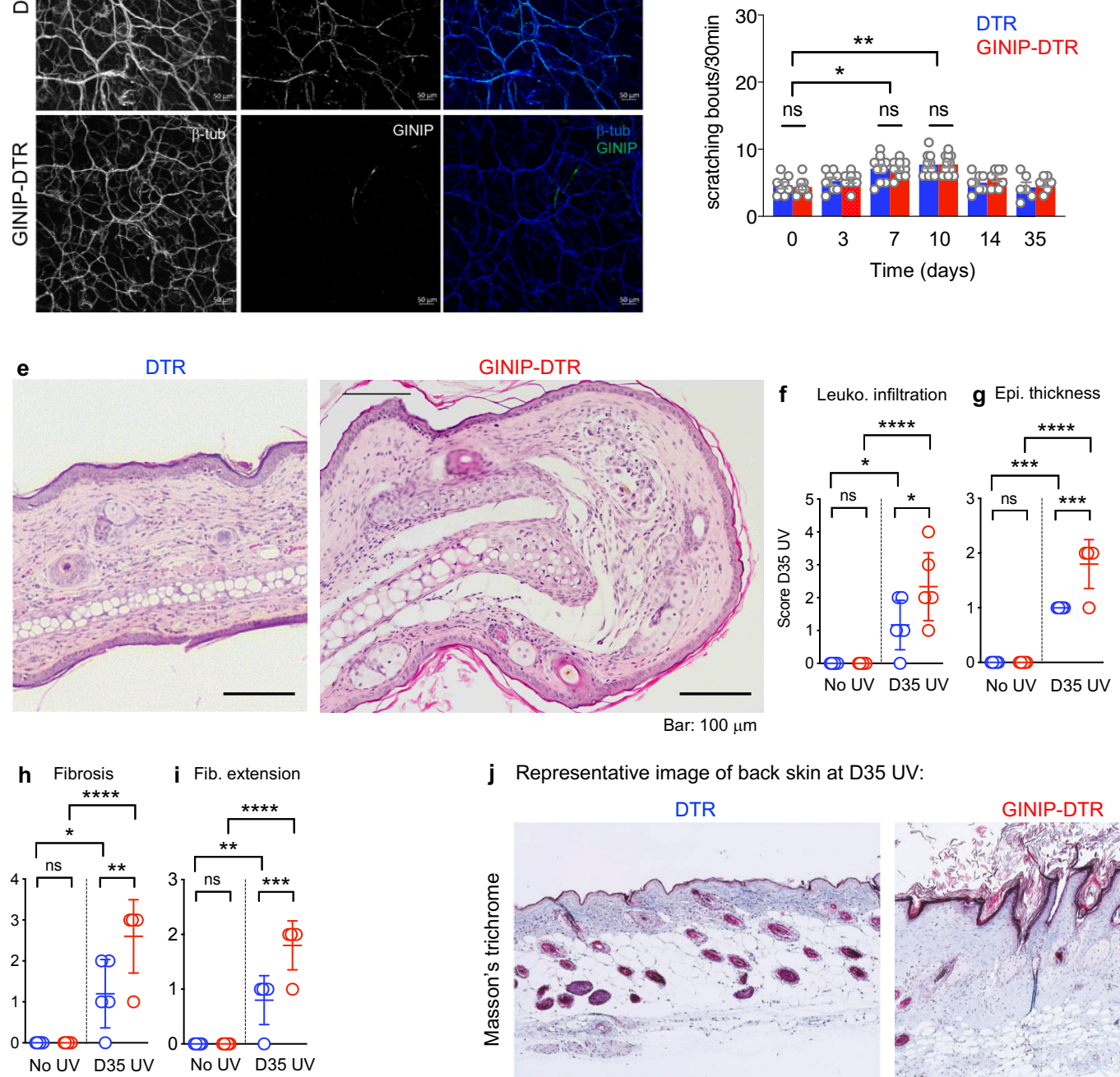

j Representative image of back skin at D35 UV:

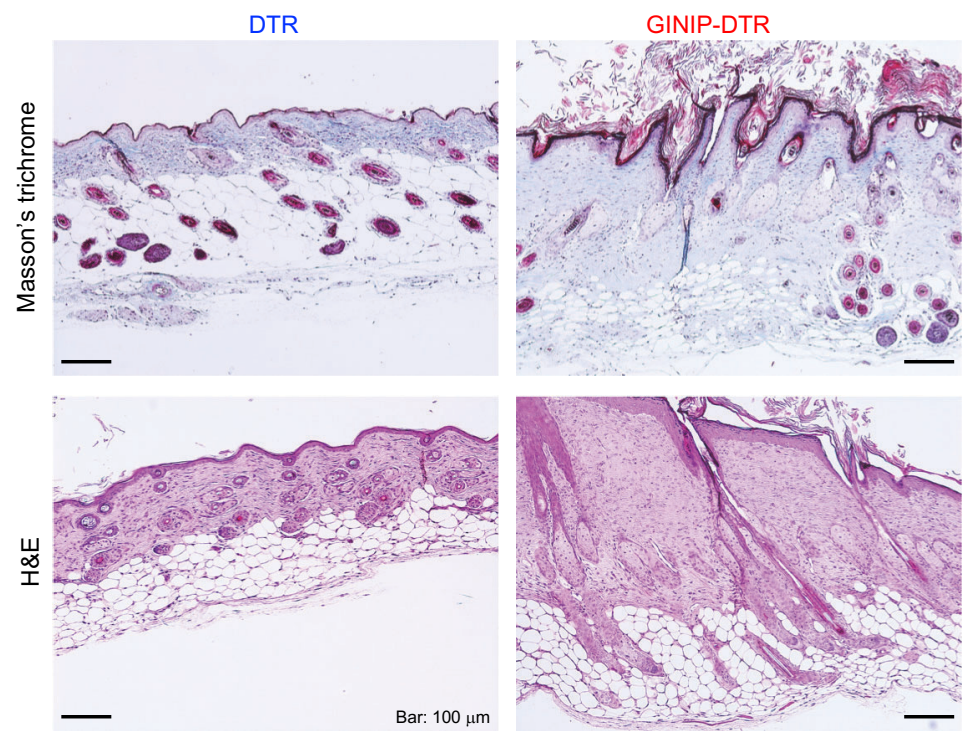


a Gating strategy for skin immune cell subsets:

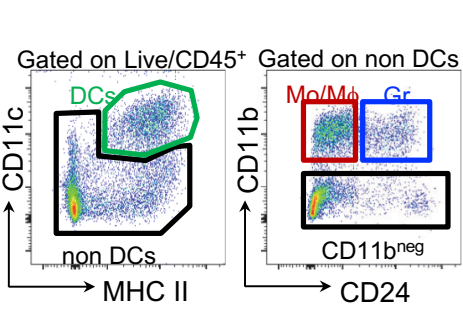

b

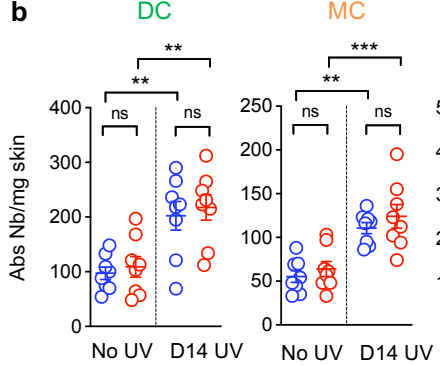

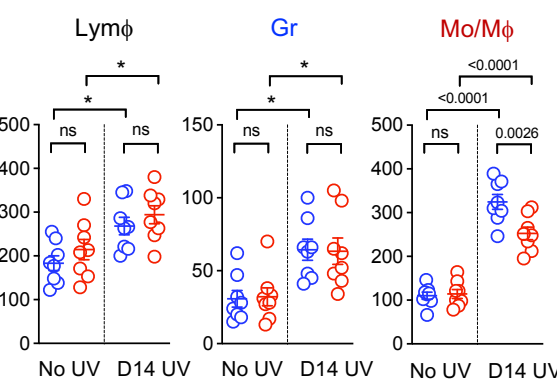

C Gating strategy for Langerhans cells (LCs):
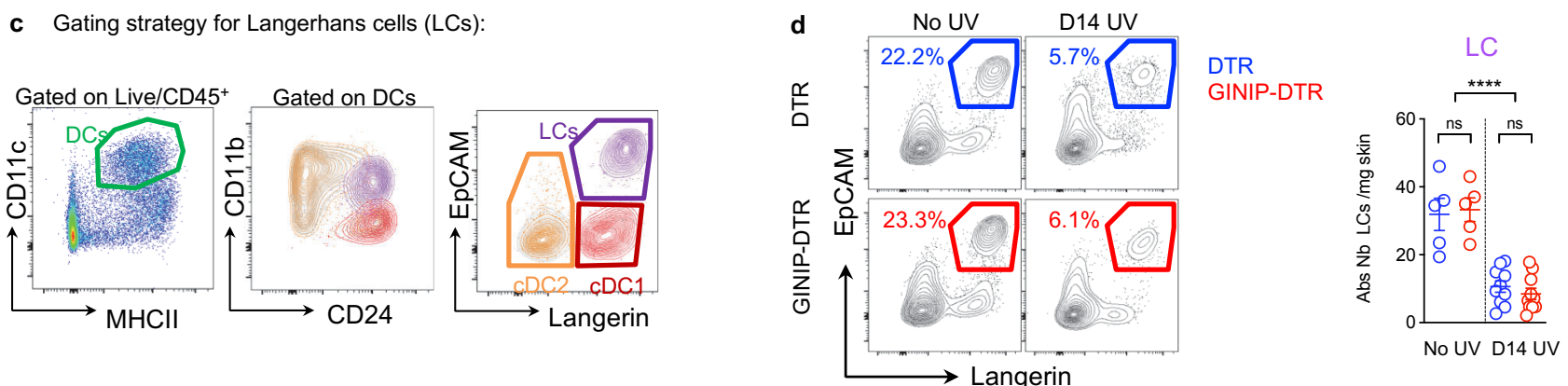

e Langerhans cells are depleted by UV irradiation:

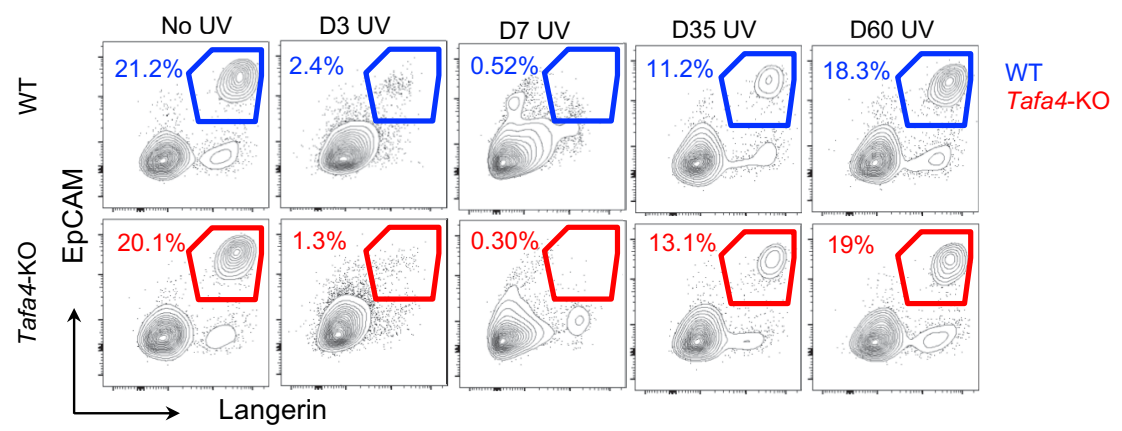

f DTR expression in GINIP-DTR mice:
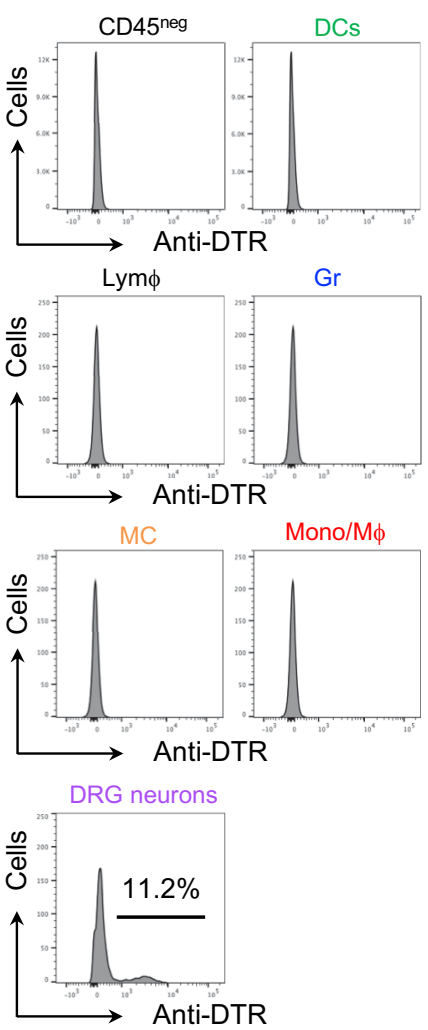

g $\mathrm{CD} 206^{+}$dermal macrophages are near GINIP ${ }^{+}$sensory neuron
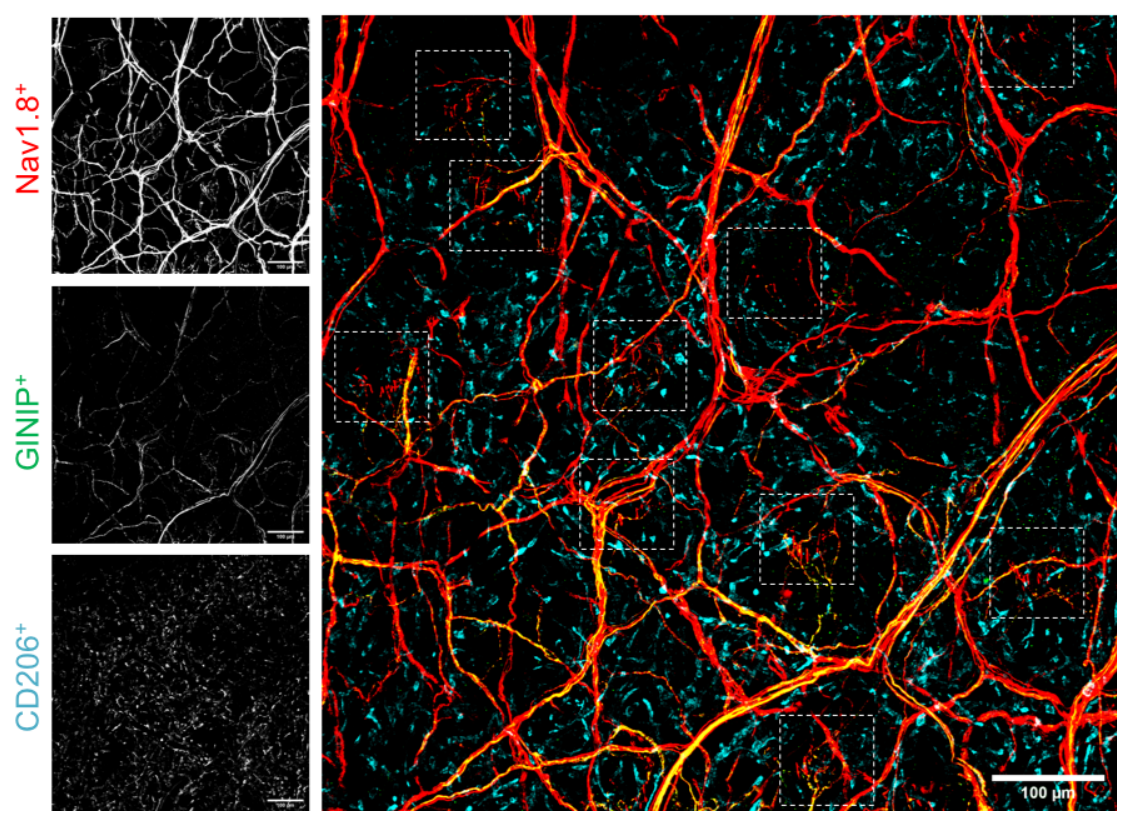

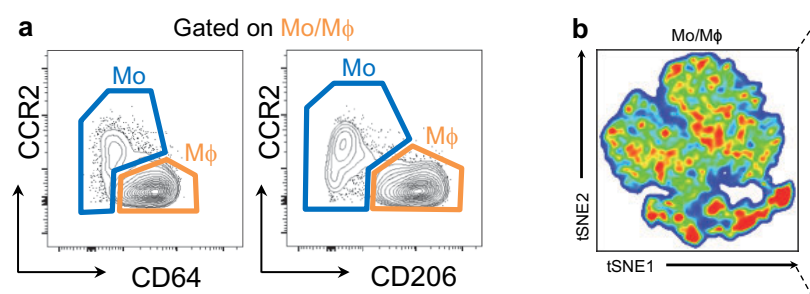

Gated on Mo Gated on Ly6Clo
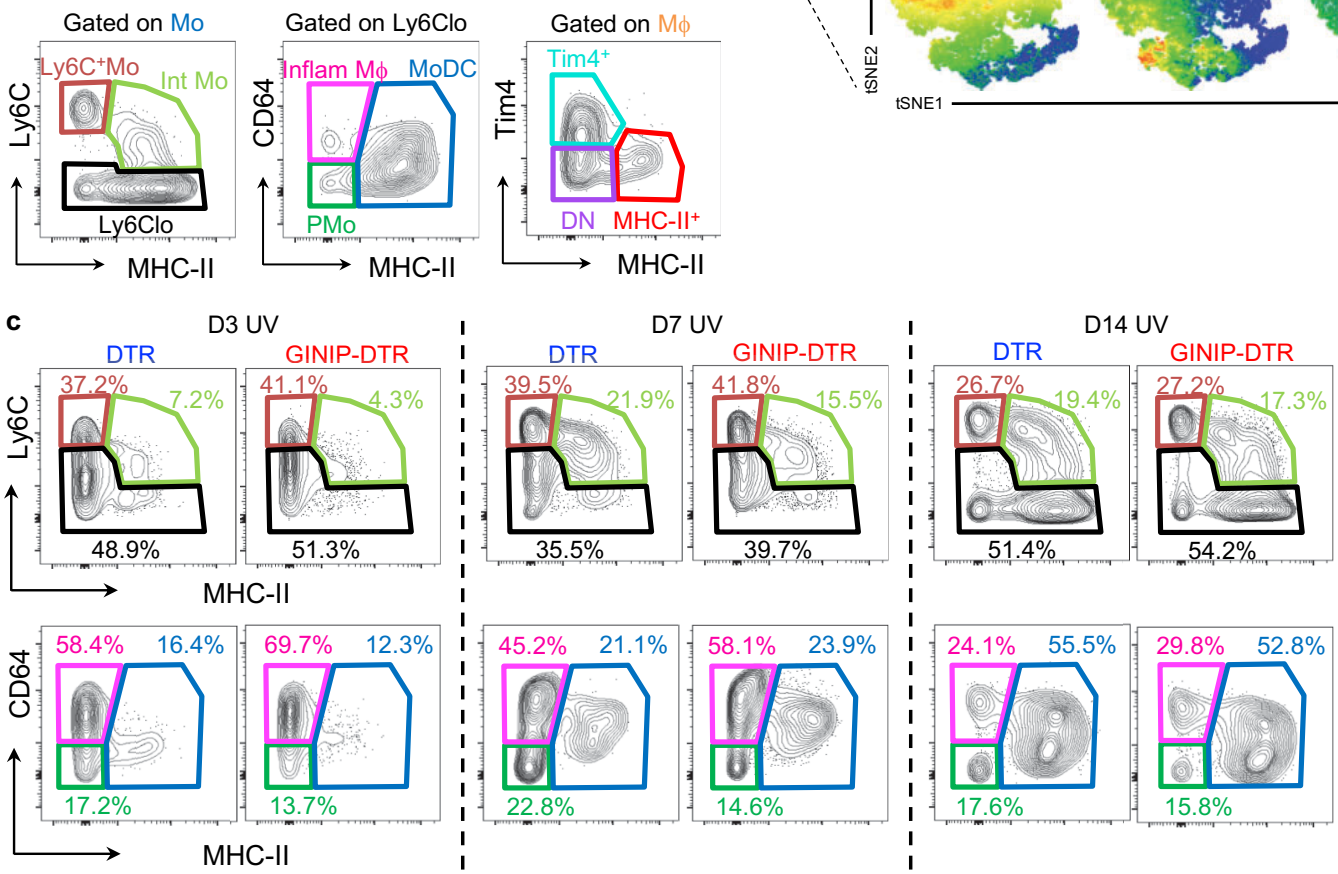

d Ly6C+ Monocytes (Ly6C+ Mo)
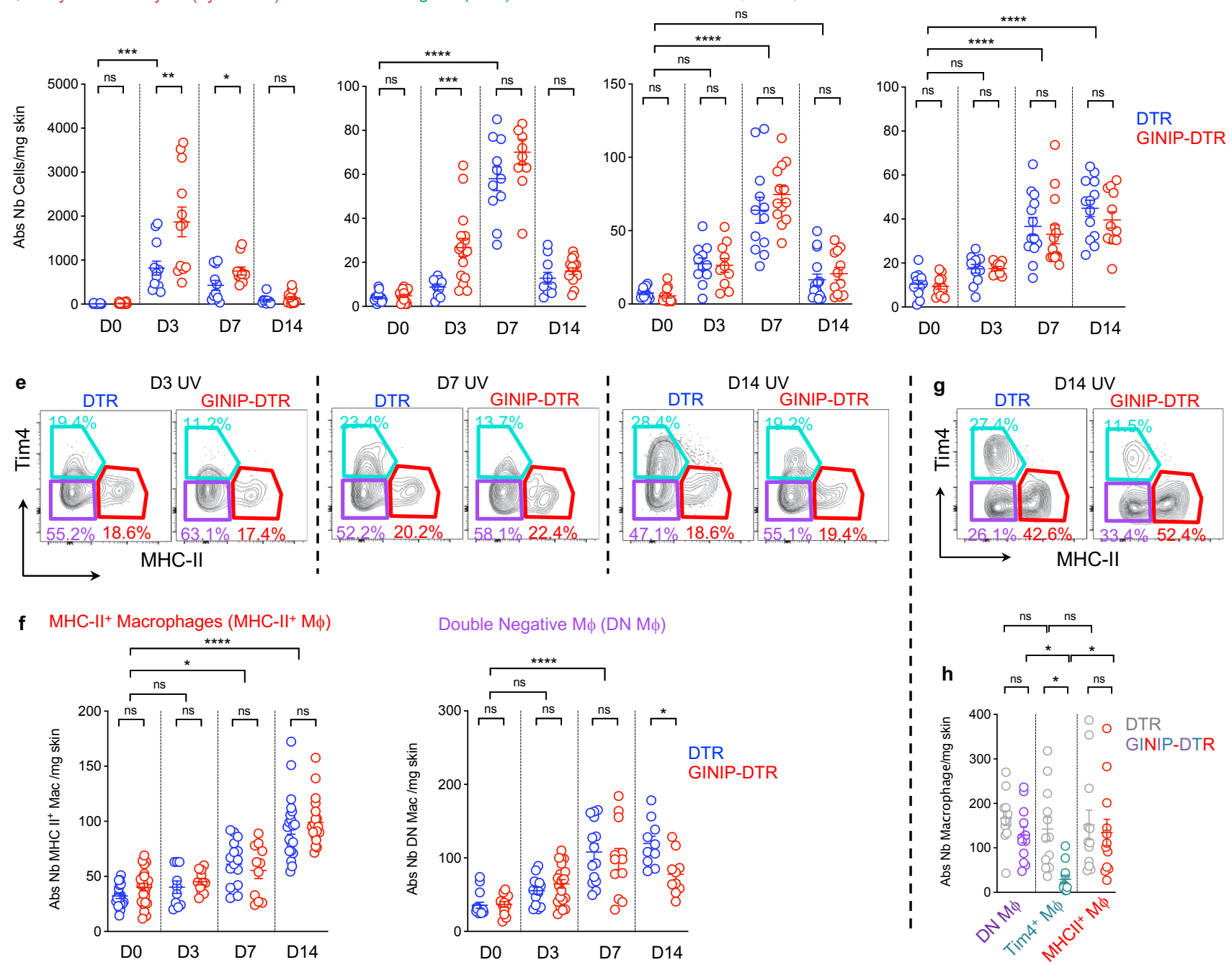

Double Negative M $\phi(D N M \phi)$

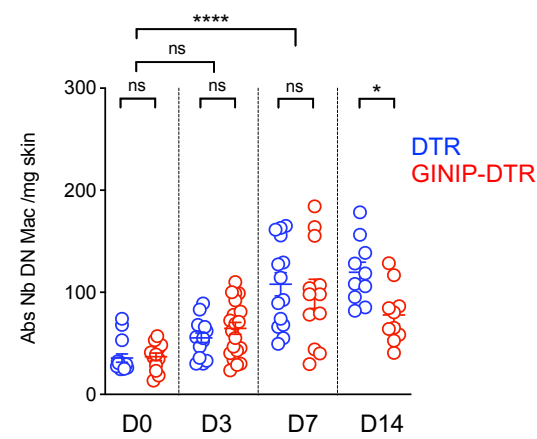




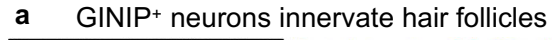

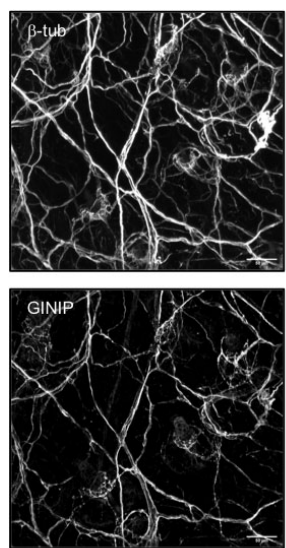

c

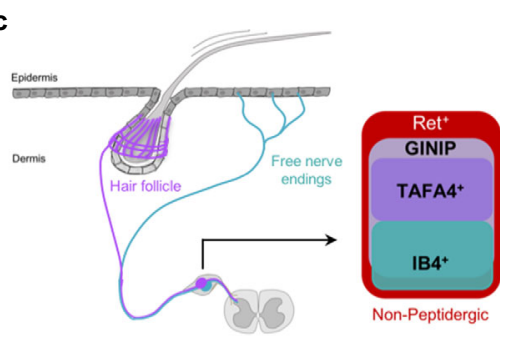

e
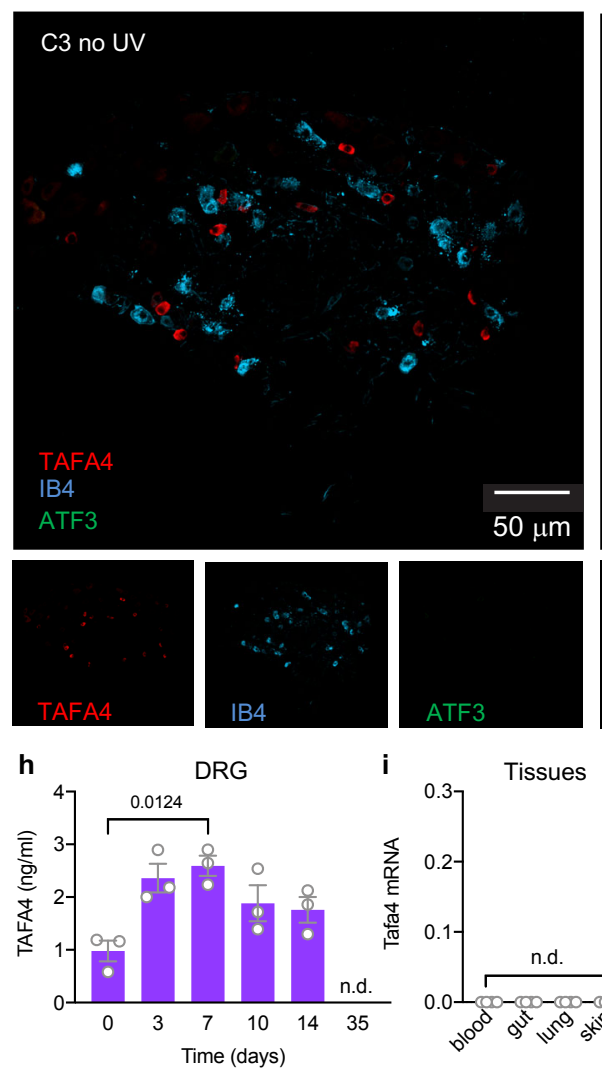

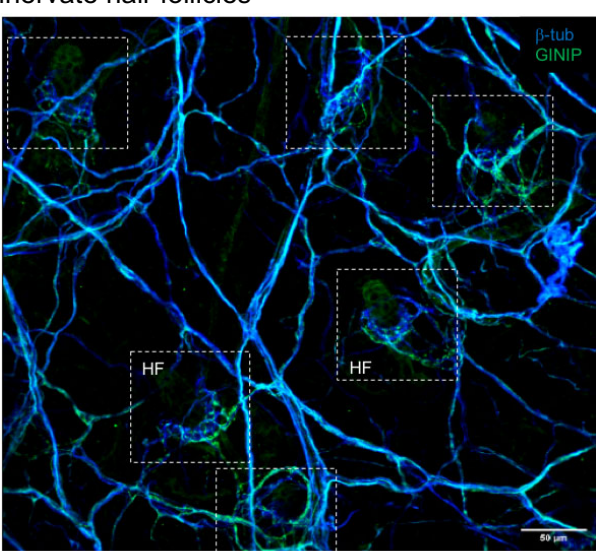

d

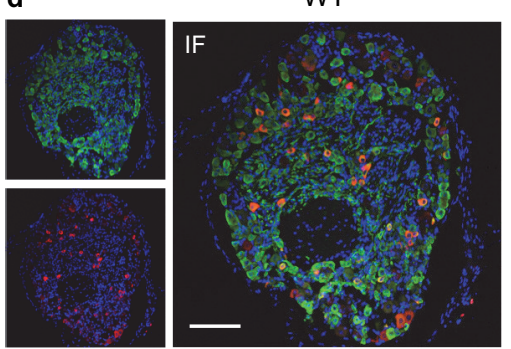

C3 D3 UV

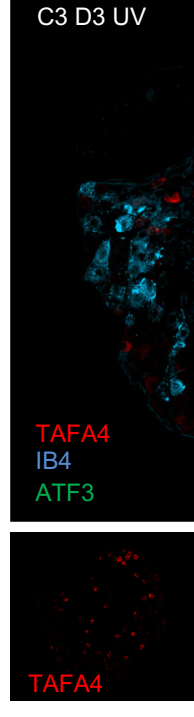

j

b

GINIP $^{+}$neurons include $\mathrm{TH}^{+} \mathrm{C}-\mathrm{LTMR}$

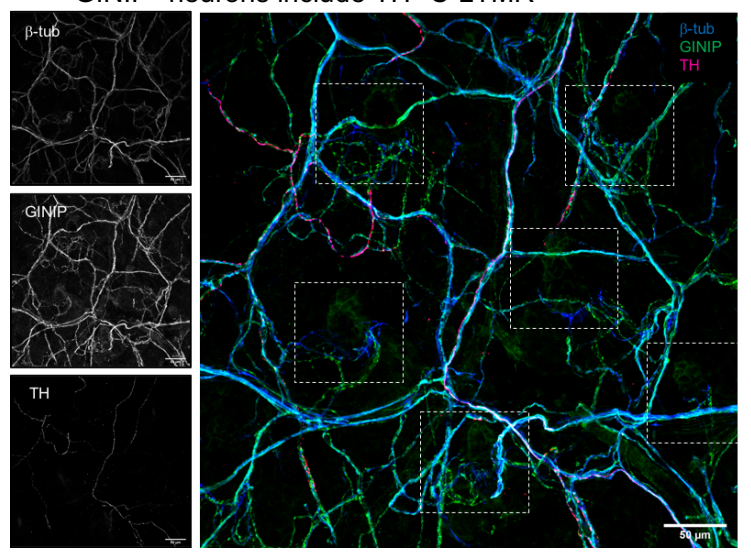

Tafa4-KO
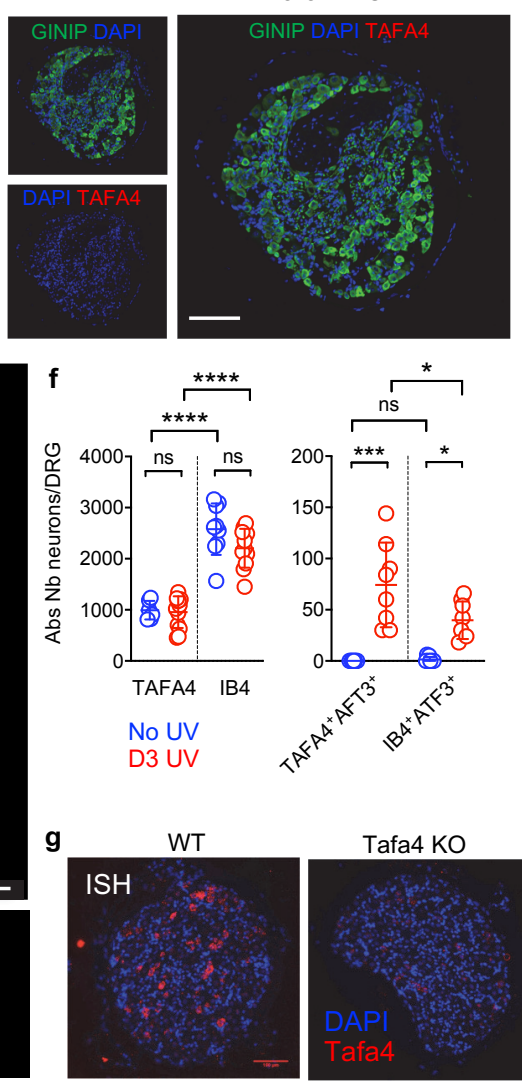

DRG

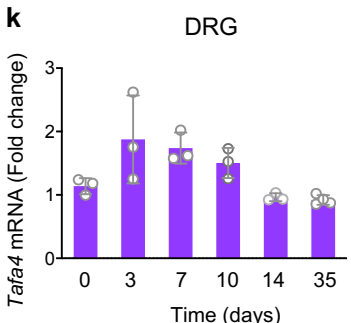

Tafa4 KO
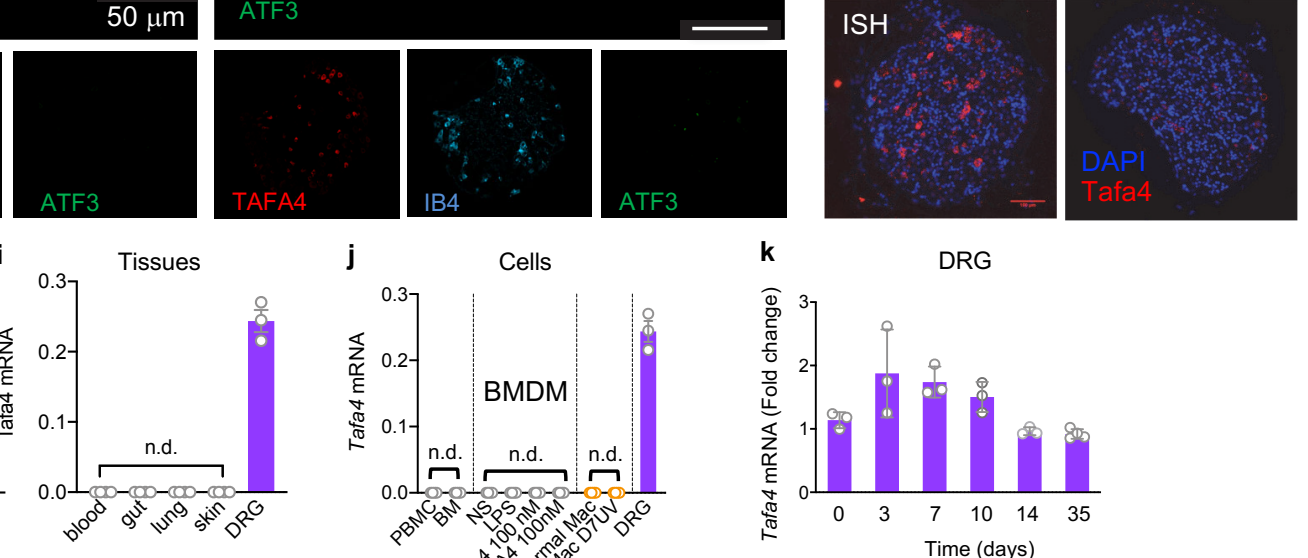

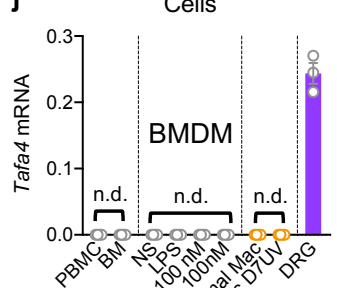

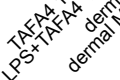



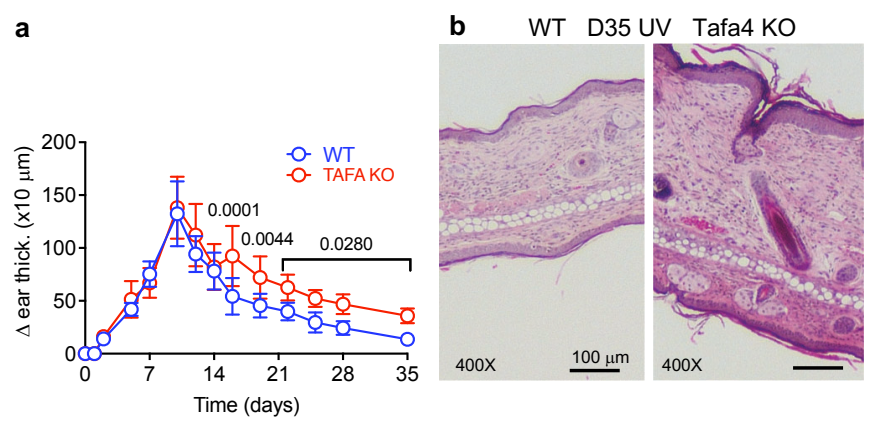

d

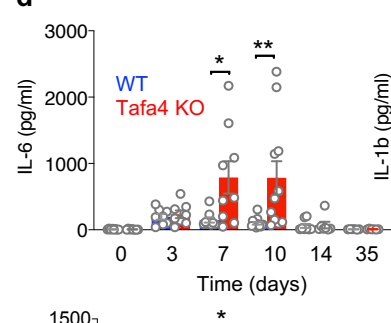

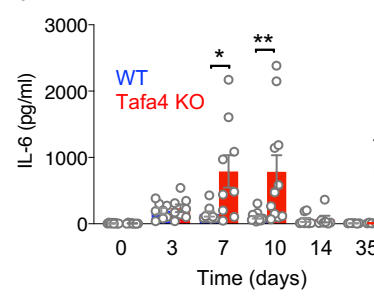

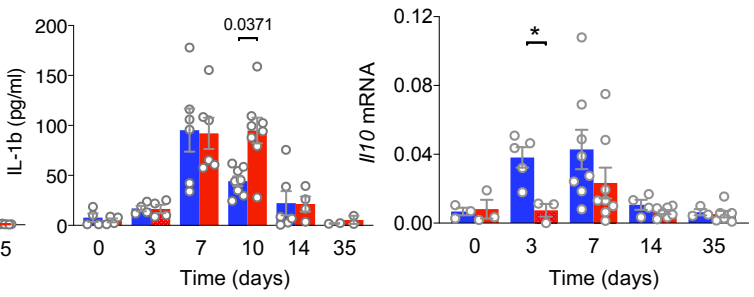

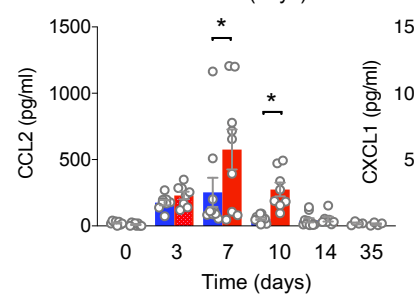
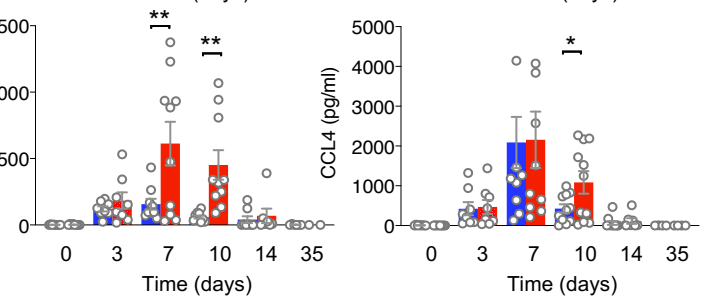
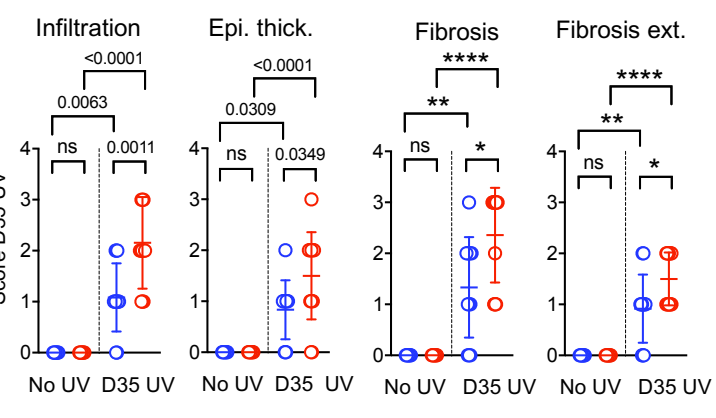

Tot. Fibrosis Path. score

c WT Tafa4 KO

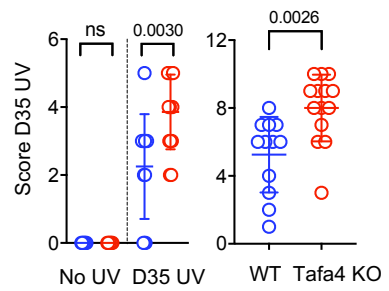

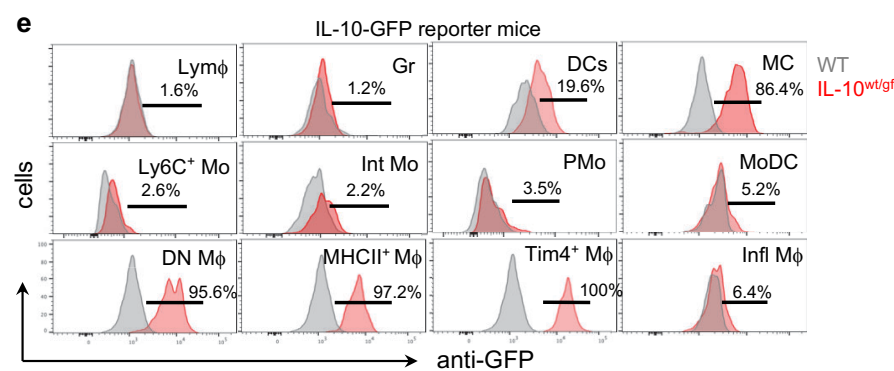

f Intracellular IL-10 staining compared to IL-10 KO mice

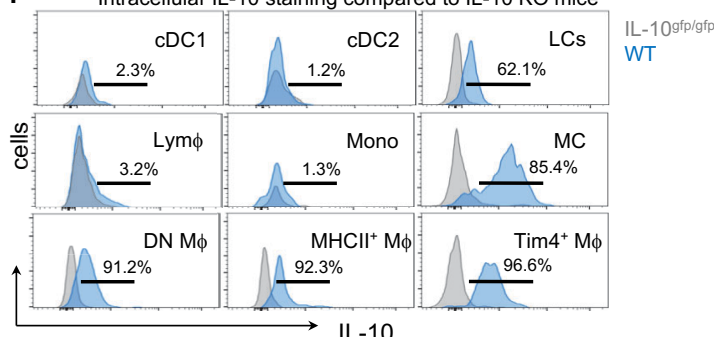

g Tregs analysis :

Gated on CD11 $b^{\text {neg }}$, non-Mast Lym $\phi$ cells, then on CD $4^{+} T C R \beta^{+}$
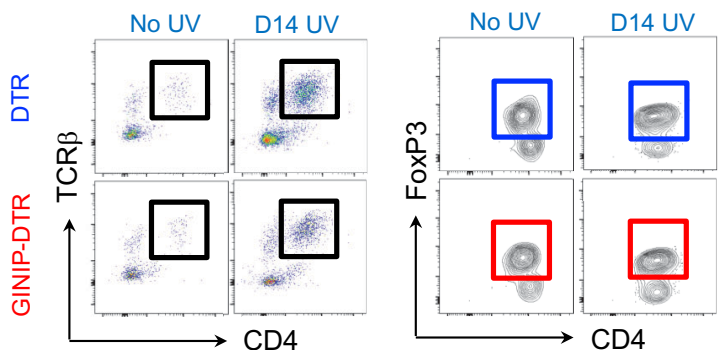

h
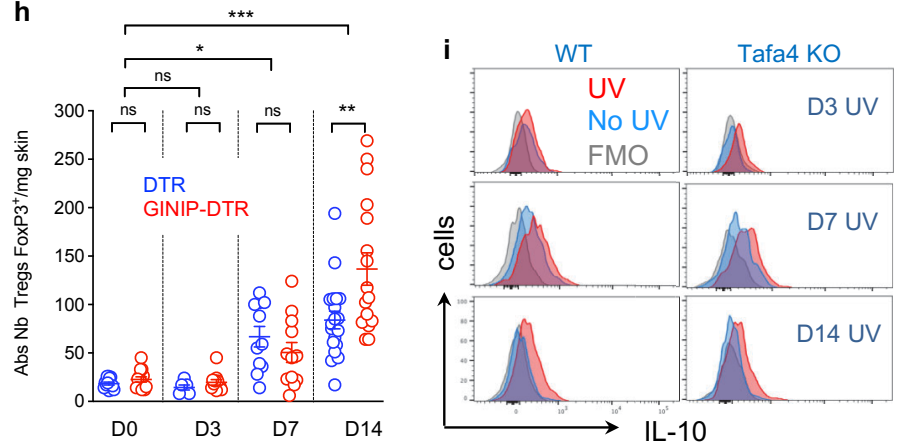

j Mast cell analysis

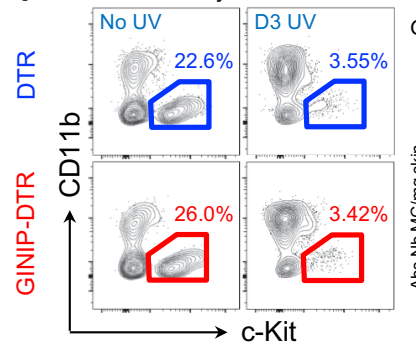

Gated on non DCs, CD24 $4^{\text {neg }}$
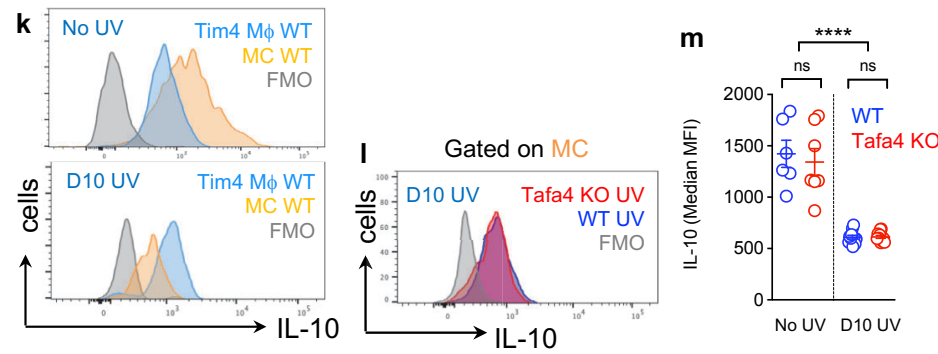

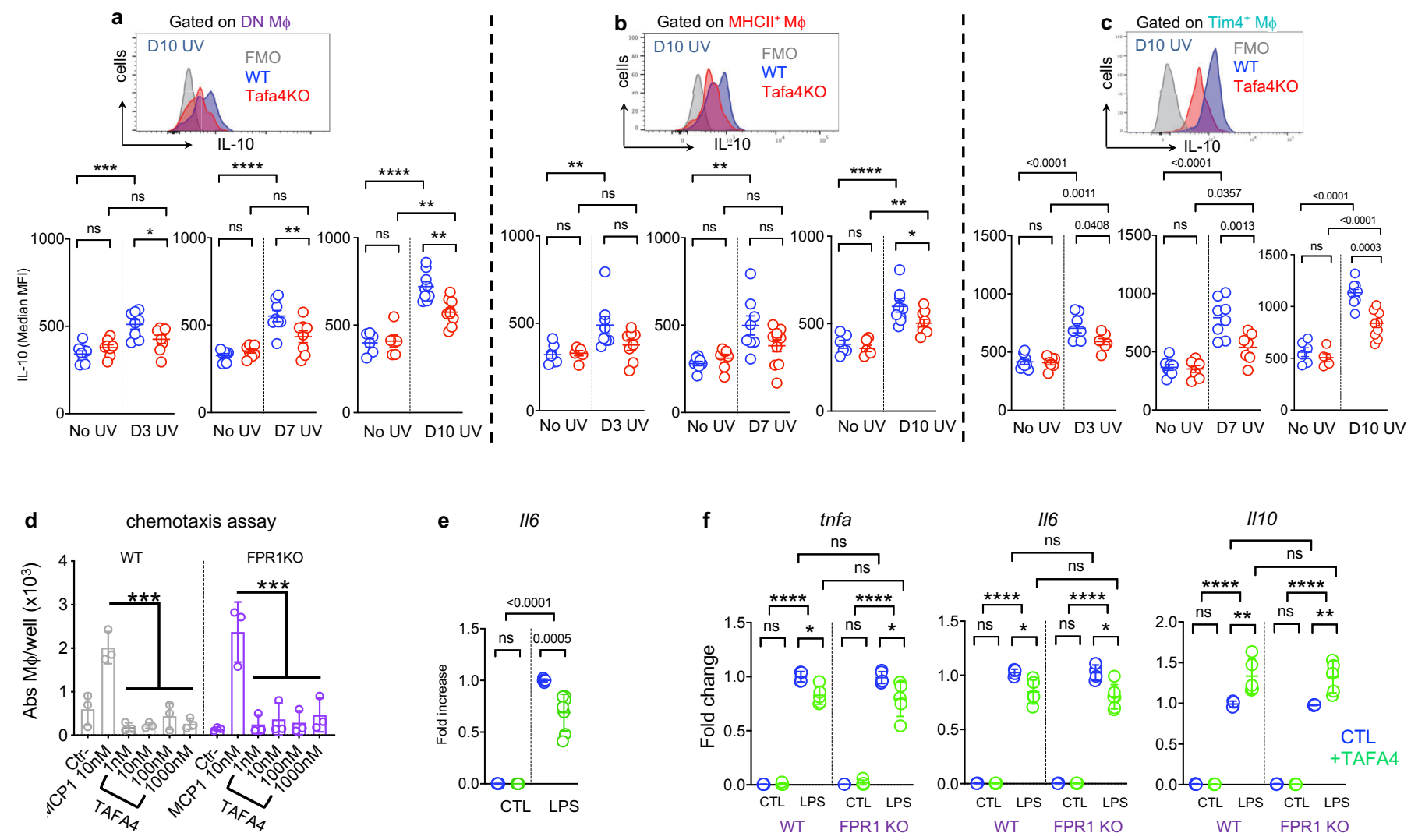

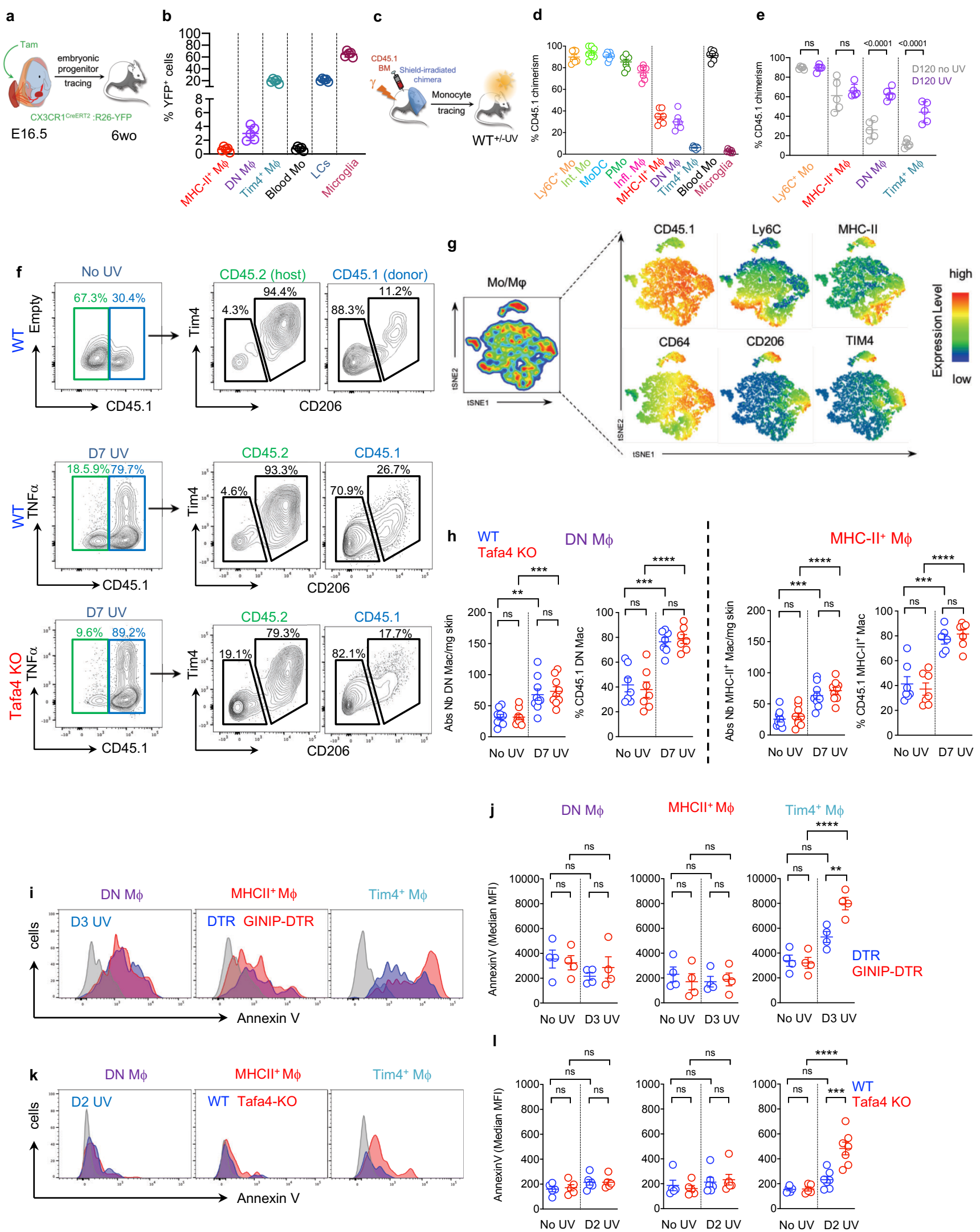


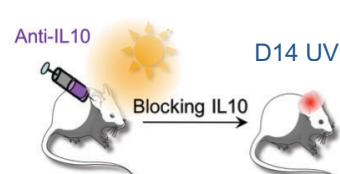

WT

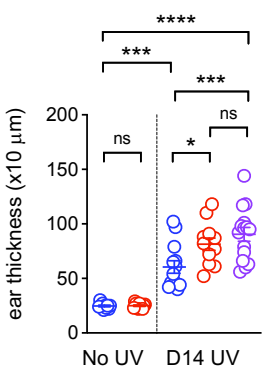

d

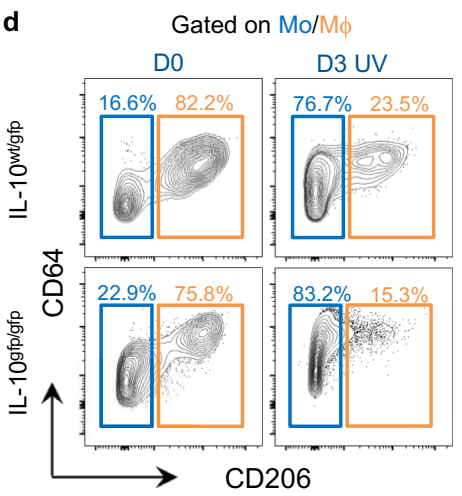

$\operatorname{Tim}^{+} \mathrm{M} \phi$

D7 UV

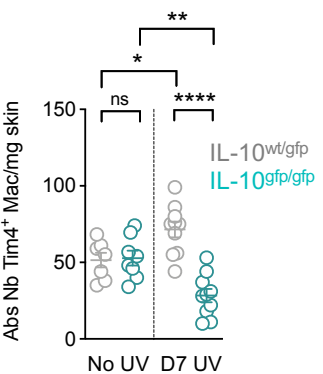

g

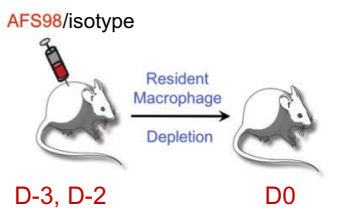

WT/Tafa4 KO

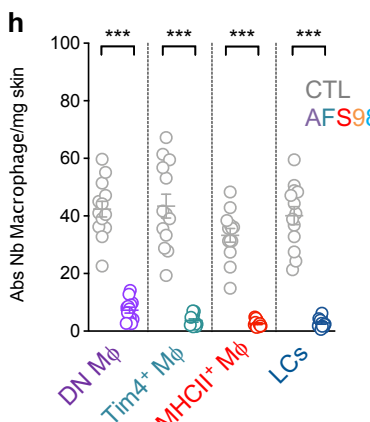

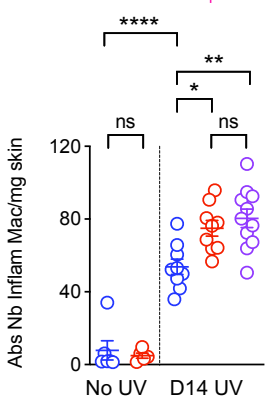
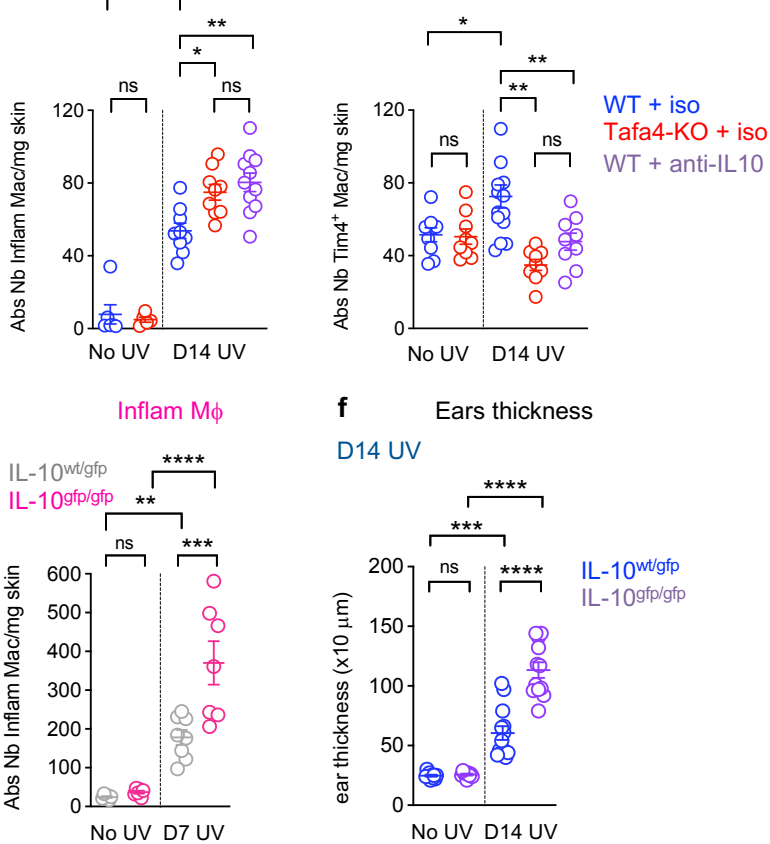

f Ears thickness

D14 UV

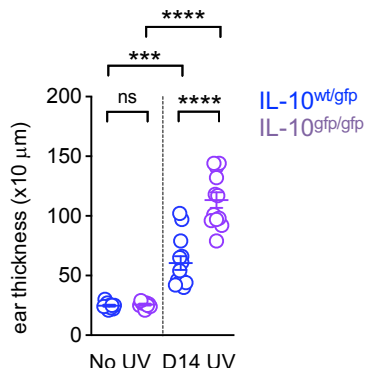

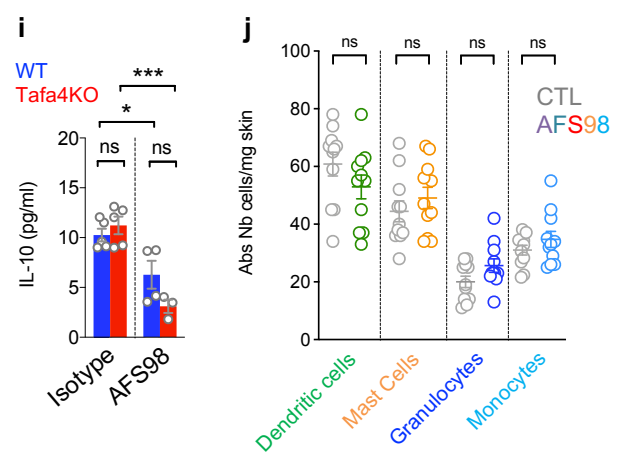

k Depleted mice (AFS98), no UV, Gated on Mo/M
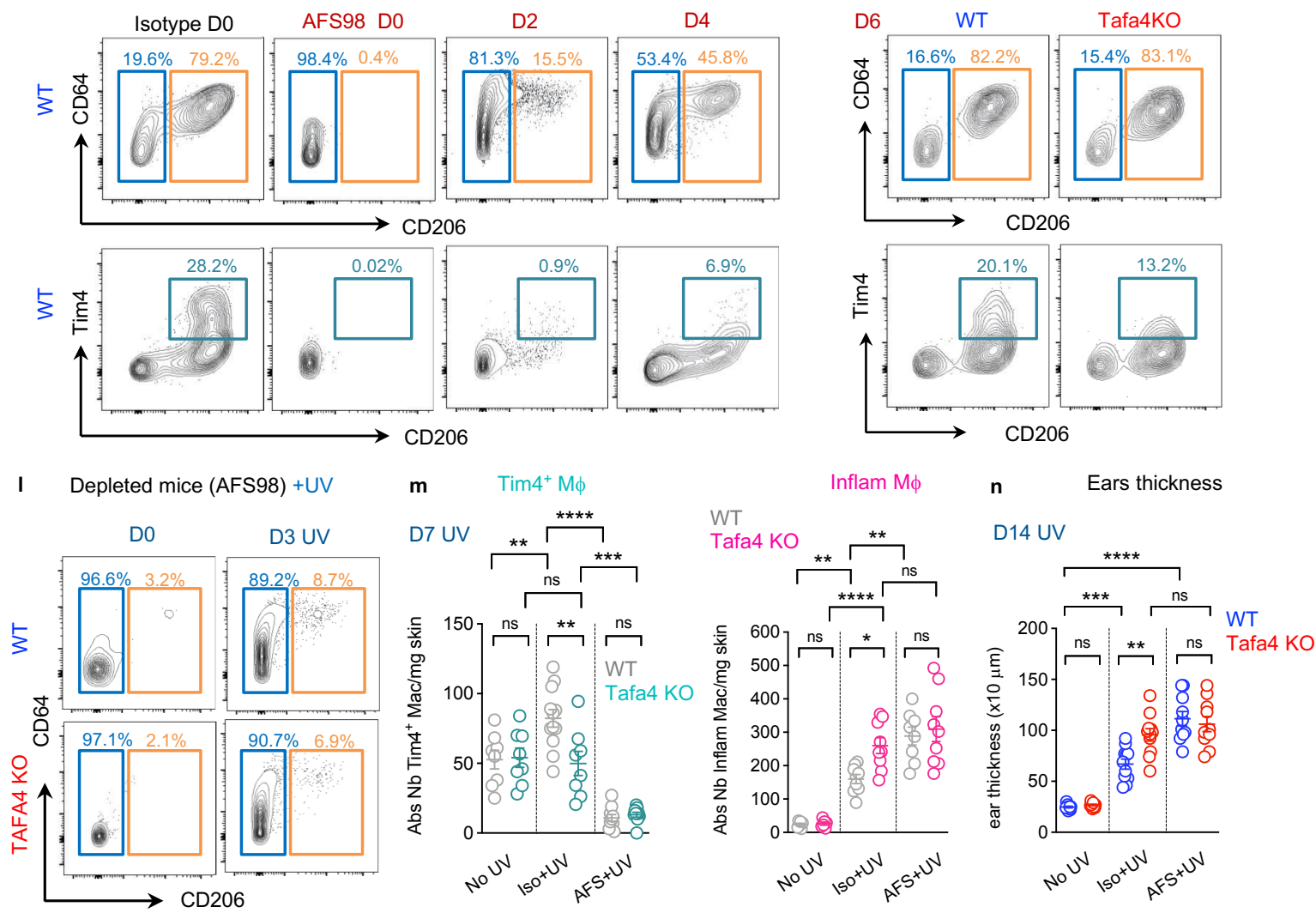
a

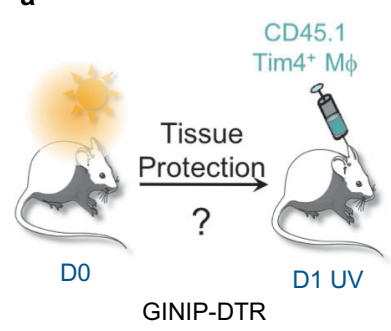

b Gated in CD45.1+CD11b+CD24-Ly6G

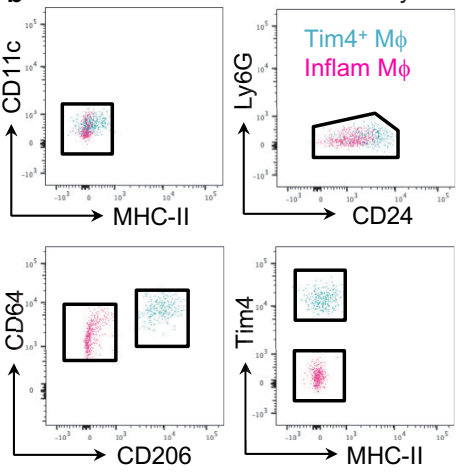

c Ear thickness

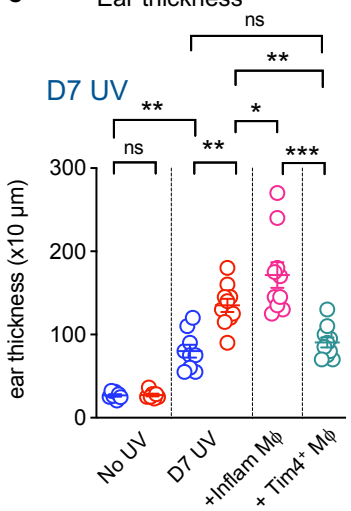

d

-DTR

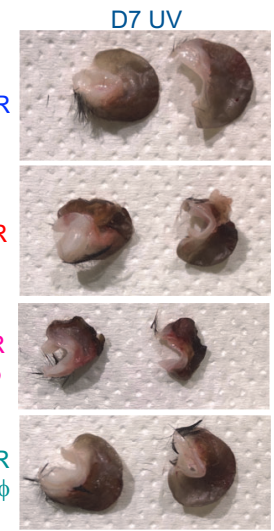

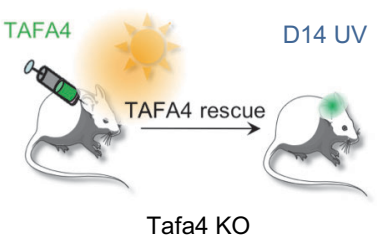

f $\quad$ Gated on $\mathrm{Tim}^{+} \mathrm{M} \phi$

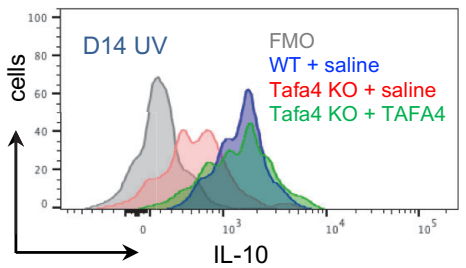

h IMQ-induced psoriatic lesion

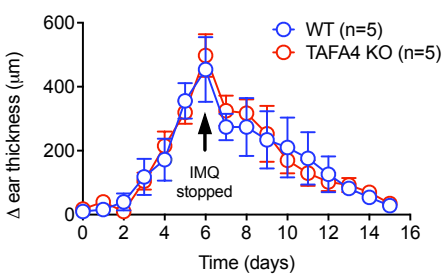

\section{g Model}

1) Role of TAFA4 during the Inflammatory phase

2) Role of TAFA4 during the Resolution phase

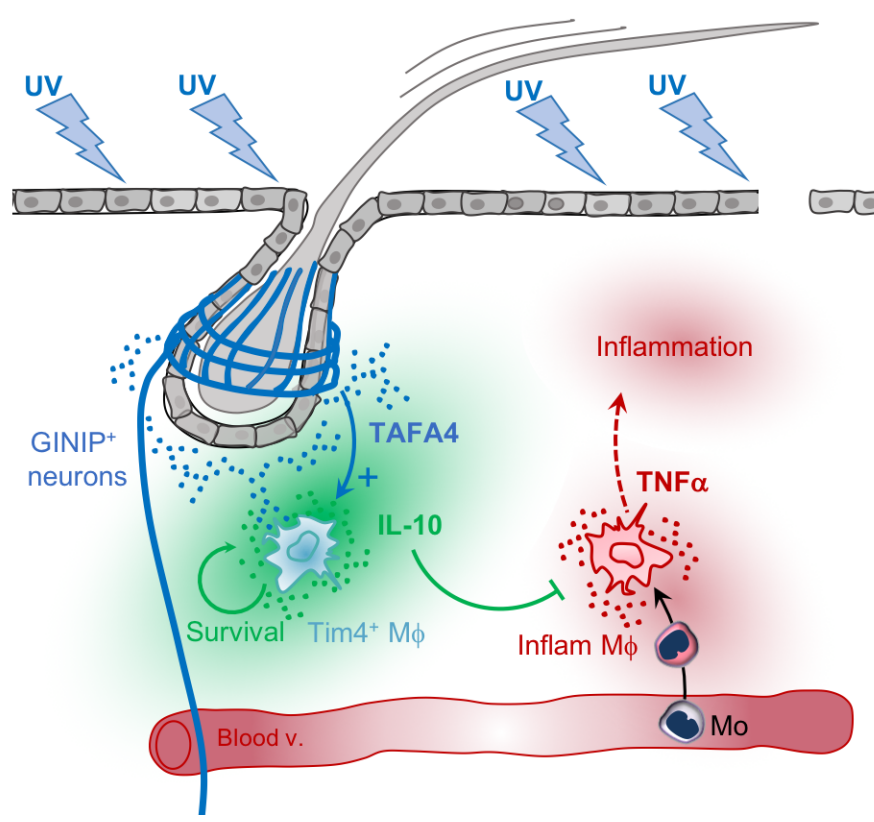

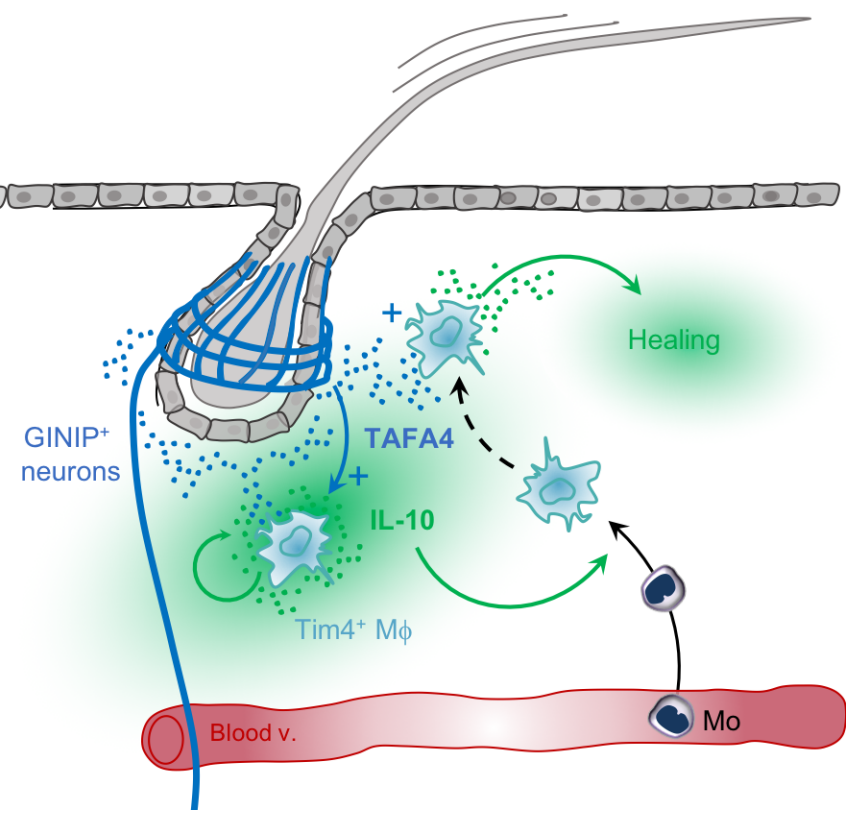

